\title{
Widely applicable periodicity results for higher order difference equations
}

\author{
István Győri, László Horváth* \\ Department of Mathematics \\ University of Pannonia \\ 8200 Veszprém, Egyetem u. 10., Hungary \\ E-mail: gyori@almos.uni-pannon.hu \\ lhorvath@almos.uni-pannon.hu \\ This paper is dedicated to Gerry Ladas on the occasion of his
retirement.
}

\begin{abstract}
In this paper we study the periodicity of higher order nonlinear equations. They are defined by a recursion which is generated by a mapping $h: X^{s} \rightarrow X$, where $X$ is a state set. Our main objective is to prove sharp conditions for the global periodicity of our equations assuming the weakest possible assumptions on the state set $X$. As an application of our general algebraic-like conditions we prove a new linearized global periodicity theorem assuming that $X$ is a normed space. We needed a new proof-technique since in the infinite dimensional case the Jacobian does not exist. We give new necessary and/or sufficient conditions as well as new examples for global periodicity, for instance whenever the state set $X$ is a group.

Keywords: difference equation, periodic solutions, global periodicity, linearized periodicity, generalized Lozi equation, periodicity over a group

2010 MSC: 39A10, 39A23
\end{abstract}

\section{Introduction}

A sequence $(x(n))$ is periodic if $x(n+p)=x(n)$ for some positive integer $p$ and all $n$. Periodic sequences arise naturally, for instance, in mathematical biology [25], communication technology [30] and [31], and in geometry and arithmetic in the study of modal theory [4]. In these cases one computes the period $p$ of the sequence in question.

\footnotetext{
*Corresponding author

E-mail address: lhorvath@almos.uni-pannon.hu
} 
It is said that a sequence $(x(n))_{n>-s}$ of elements of a fixed nonempty set $X$ is recursively generated if there exist functions $h_{n}: D_{h_{n}}\left(\subset X^{s}\right) \rightarrow X$ such that

$$
x(n)=h_{n}(x(n-1), \ldots, x(n-s)), \quad n \geq 0,
$$

where $s \geq 1$ is a given integer. In this case $(x(n-1), \ldots, x(n-s)) \in D_{h_{n}}$ for any $n \geq 0$. We also will say that the sequence $(x(n))_{n \geq-s}$ is a solution of the difference equation (1). It is clear that the solutions of (1) are uniquely determined by their initial values

$$
x(n)=\varphi(n), \quad-s \leq n \leq-1,
$$

where $\varphi=(\varphi(-s), \ldots, \varphi(-1)) \in D_{a d}\left(\subset X^{s}\right)$. Here $D_{a d}$ denotes the set of all admissible initial vectors which means that the recursion (1) determined by $\varphi \in D_{a d}$ is well defined for all $n \geq 0$. For $\varphi \in D_{a d}$ the unique solution of (1) and (2) is denoted by $x(\varphi)=(x(\varphi)(n))_{n \geq-s}$.

In this paper our main results are related to the investigation of the global periodicity property of higher order difference equations on a set $A \subset D_{a d}$.

Definition 1 Consider equation (1), and let $\varphi=(\varphi(-s), \ldots, \varphi(-1)) \in D_{a d}$ be a given initial vector.

(a) The solution $x(\varphi)$ of (1) and (2) is called periodic if there exists a positive integer $p$ such that $x(\varphi)(n+p)=x(\varphi)(n)$ for all $n \geq-s$. In this case we say that $x(\varphi)$ is p-periodic.

(b) $\varphi$ is said to be a p-periodic initial vector of (1) if the solution $x(\varphi)$ of (1) and (2) is p-periodic.

(c) We say that $p$ is the prime period of the solution $x(\varphi)$ of (1) and (2) if it is p-periodic and $p$ is the smallest positive integer having this property.

(d) We say that equation (1) is globally p-periodic on the set $A \subset D_{a d}$ if every solution of (1) determined by an initial value from $A$ is p-periodic. If $A=D_{a d}$, then we simple say that (1) is globally p-periodic.

The global periodicity property has attracted the attention of many researchers (see for example the papers [2], [3], [5], [6], [8], [9], [10], [13], [18], [19], [20], [22], [24] and [28], and the references therein).

Most of the periodicity results in the literature are classical in the sense that the investigated difference equations are scalars or finite dimensional systems, that is $X$ is either $\mathbb{R}$ or $\mathbb{R}^{d}$. At the same time there is an increasing interest in several different type of difference equations. Without seeking for completeness we mention the following subclasses:

Boolean delay equations which are novel modeling framework especially tailored for the mathematical formulation of conceptual models of systems that exhibit treshold behavior, multiple feedbacks and distinct delays (see [16] and [13]).

Periodic integer solutions are studied in [17] for some second order difference equations. It is interesting to note that in some subclass of such difference equations, any solution which is not eventually periodic must be unbounded, so 
the real problem is to classify the periodic solutions which appears to be very difficult (see [11], [17] and [23]).

Motivated, for instance, by the control theory and the neural network models, there is a growing interest in difference equations with piecewise linear right hand side (see [2] and [7]). An interesting subclass of these equations are the Lozi equation and its several generalizations (see [1] and [7]). In this case the periodicity property of the solutions should be restricted to some set $D^{d} \subset \mathbb{R}^{d}$, where $D$ is a nondegenerate interval of real numbers.

Difference equations in abstract (infinite dimensional) spaces are also investigated but the numbers of such papers comparing to the classical case is small. In particular, the paper [15] should be mentioned, in which non-positive of abstract difference equations are examined.

Higher order difference equations are discussed in rings and also in groups in [26]. The author gives semiconjugate factorizations which are applied for second order recurrences that define modified Bessel functions or Chebysev polinomials, but with arbitrary initial functions.

The above discussions show that the investigation of the difference equation (1) is interesting not only in a finite dimensional vector space $X$ but for the cases when $X$ is an infinite dimensional space, a group, a ring, or only a simple set without any structure.

Motivated by the above discussion, the objective of this paper is to study the periodicity of equation (1) assuming the weakest possible condition on $X$.

Section 2 is devoted to the main results and it is subdivided into two parts. Subsection 2.1 contains powerful sufficient and/or necessary conditions for global $p$-periodicity of the autonomous form of equation (1)

$$
x(n)=h(x(n-1), \ldots, x(n-s)), \quad n \geq 0,
$$

where $h: D_{h}\left(\subset X^{s}\right) \rightarrow X$. The formulation of the results is based on a composit function of which derivation depends on the situations $p<s$ or $p=s$ or $p>s$. The applicability of these results are illustrated in Section 4 . They also play an important role in the proof of our linearized global periodicity results which are stated in Subsection 2.2.

Global periodicity of difference equations in an Euclidean space via linearization has been considered by many authors, see e.g. [28], [6], [9], [10], [22], [5], [2], [24], [3] and the references therein. In these papers the authors consider those recursions whose respective generators have a power series expansion, but at least continuous partial derivatives about a fixed point. In particular, it makes sense to consider the Jacobian of $h$ in (3) at that point (i.e. linear part of $h$ with respect to its power series expansion). They show that one can determine the period of the nonlinear system by merely computing the period of the Jacobian at the fixed point.

It happens that some features of the finite dimensional recursion remain intact in the infinite dimensional cases.

What remain intact in abstract case are: First of all the ability to compute the so called Fréchet derivative of the right hand side function $h$ of (3) only at a 
fixed point assuming that it exists in a real normed space $X$. Second, the ability to prove that in the above case the period of the nonlinear equation is exactly equal to the period of the associated linearized equation as it is known in finite dimensional case. Of course, a globally periodic linearized equation does not imply a globally periodic nonlinear equation (see [6]).

It is worth to note that the Fréchet derivative in finite dimensional spaces is the usual derivative. In particular, it is represented in coordinates by the Jacobian matrix.

In our linearized periodicity theorem (Theorem 12 in Section 2.2) we assume only three natural assumptions: The basic set $X$ is a real normed space, equation (3) has an equlibrium, and the nonlinear mapping $h$ on the right hand side of (3) is Fréchet differentiable at the equlibrium point. In most of the former linearized results it is assumed that $h$ is given by a power series or at least it is continuously differentiable in a neighbourhoud of the equlibrium. So our differentiability condition is weaker.

Because of our assumptions, we needed a new proof technique which is different from those utilized in the above mentioned former related papers and which are using Jacobian in finite dimensional case. In spite of the abstract case setting our proof, presented in Section 5, is elementary.

The following statement is a simple corollary of our linearized periodicity theorem:

Proposition 2 If $X$ is a real normed space, then it does not exist any continuous multilinear map $h: X^{s} \rightarrow X(s \geq 2)$ for which equation (3) is globally periodic.

For the convenience of the reader, we recall that the map $h: X^{s} \rightarrow X$ is multilinear if it has the following property: for each $i$, if all of the variables but $x_{i}$ are held constant, then the function $x_{i} \rightarrow h\left(x_{1}, \ldots, x_{i}, \ldots, x_{s}\right)\left(x_{i} \in X\right)$ is a linear. A well known bilinear but not liniar map is the cross product of two vectors in $\mathbb{R}^{3}$.

The proof of the above proposition is based on the fact that every continuous multilinear map is Fréchet differentiable and the Fréchet derivative $d h$ is linear (see [14]). On the other hand $0 \in X$ is an equlibrium of equation (3) and thus the linearized equation of (3) around 0 is

$$
x(n)=0, \quad n \geq 0,
$$

which is not globally $p$-periodic. We now deduce the result from our Theorem 12 in Subsection 2.2 .

In Section 3 some results are given for the existence of periodic solutions which are interesting in their own rights and they are also important in the proofs of the main theorems.

In Section 4 several illustrative examples and corollaries are given to show the effectiveness of our approaches.

The advantage of the general formulation of our global periodicity results is presented in the investigation of the following problem. 
Problem: For a fixed integer $s \geq 2$, find all the form of the map $f: X \rightarrow X$ for which the equation

$$
x(n)=f(x(n-1)) \ldots f(x(n-s)), \quad n \geq 0
$$

is globally $(s+1)$-periodic.

The above problem has been motivated by the paper [5], where a more general related problem has been solved. For some recent results in this research area see for example [29] and [9]. In fact, in the scalar case e.g. $X=\mathbb{R}$, it is proved that $(4)$ is $(s+1)$-periodic if and only if

$$
f(x)=c x^{-1}, \quad x \in \mathbb{R} \backslash\{0\},
$$

where $c$ is an arbitrary fixed nonzero constant. Motivated by this result and by the paper [26], we have proved the following result.

We consider a group $G$, where the binary operation is written multiplicatively, the identity element is denoted by $e$, and the inverse of $x \in G$ is denoted by $x^{-1}$.

Theorem 3 Let $f$ be a function from $G$ into $G$, and let $s$ be a positive integer. Equation (4) is globally $(s+1)$-periodic if and only if

$$
f(x)=\alpha x^{-1}, \quad x \in G,
$$

where $\alpha$ is a fixed element from the center

$$
\{\alpha \in G \mid \alpha x=x \alpha \text { for all } x \in G\}
$$

of $G$.

We consider two special cases of this theorem corresponding to two different matrix groups. In both cases the operation is the matrix multiplication.

$G L_{d}(\mathbb{R})$ denotes the real general linear group of degree $d$, that is the set of $d \times d$ invertible matrices.

$H_{3}(\mathbb{R})$ denotes the continuous Heisenberg group (see [27]), which is the group of $3 \times 3$ upper triangular matrices of the form

$$
\left(\begin{array}{ccc}
1 & a & c \\
0 & 1 & b \\
0 & 0 & 1
\end{array}\right), \quad a, b, c \in \mathbb{R} .
$$

The center of $G L_{d}(\mathbb{R})$ and $H_{3}(\mathbb{R})$ are

$$
\left\{a I_{d} \in \mathbb{R}^{d \times d} \mid a \in \mathbb{R} \backslash\{0\}\right\},
$$

where $I_{d}$ is the identity matrix in $\mathbb{R}^{d \times d}$, and

$$
\left\{\left(\begin{array}{ccc}
1 & 0 & z \\
0 & 1 & 0 \\
0 & 0 & 1
\end{array}\right) \in \mathbb{R}^{3 \times 3} \mid z \in \mathbb{R}\right\}
$$


respectively.

A simple corollary of Theorem 3 we get the next proposition for the matrix difference equation

$$
M(n)=f(M(n-1)) \ldots f(M(n-s)), \quad n \geq 0 .
$$

Corollary 4 Let $s$ be a positive integer.

(a) Equation (5) is globally $(s+1)$-periodic for the mapping $f: G L_{d}(\mathbb{R}) \rightarrow$ $G L_{d}(\mathbb{R})$ if and only if

$$
f(M)=a M^{-1}, \quad M \in G L_{d}(\mathbb{R}),
$$

where $a \in \mathbb{R} \backslash\{0\}$.

(b) Equation (5) is globally $(s+1)$-periodic for the mapping $f: H_{3}(\mathbb{R}) \rightarrow$ $\mathrm{H}_{3}(\mathbb{R})$ if and only if

$$
f(M)=C M^{-1}, \quad M \in H_{d}(\mathbb{R}),
$$

where $C$ is from the center of $H_{3}(\mathbb{R})$.

The proofs of our results are presented in Section 5.

\section{Main results}

\subsection{Periodicity of all solutions of higher order difference systems}

We consider equation (3)

$$
x(n)=h(x(n-1), \ldots, x(n-s)), \quad n \geq 0,
$$

where $s \geq 1$ is a given integer, and $h: D_{h}\left(\subset X^{s}\right) \rightarrow X$.

We shall continue to denote by $D_{a d}$ the set of all admissible initial vectors.

For every set $H \subset X^{s}$ let

$$
\hat{H}:=\left\{\left(y_{1}, \ldots, y_{s}\right) \in X^{s} \mid\left(y_{s}, \ldots, y_{1}\right) \in H\right\} .
$$

We need the following recursion: For $\left(y_{s}, y_{s-1}, \ldots, y_{1}\right) \in D_{a d}$ let

$$
\begin{aligned}
f_{-s}\left(y_{1}, y_{2}, \ldots, y_{s}\right) & :=y_{s} \\
f_{-s+1}\left(y_{1}, y_{2}, \ldots, y_{s}\right) & :=y_{s-1}, \\
\vdots & \\
f_{-1}\left(y_{1}, y_{2}, \ldots, y_{s}\right) & :=y_{1}
\end{aligned}
$$

and define

$$
f_{i}:=h \circ\left(f_{i-1}, f_{i-2}, \ldots, f_{i-s}\right), \quad i \geq 0 .
$$


It follows from the definition of the set $D_{a d}$ that the previous recursion is well defined and

$$
D_{f_{i}}=\hat{D}_{a d}, \quad i \geq 0 .
$$

If $\varphi:=\left(y_{s}, y_{s-1}, \ldots, y_{1}\right) \in D_{a d}$, then by $(7)$ and $(8)$

$$
f_{i}\left(y_{1}, y_{2}, \ldots, y_{s}\right)=x(\varphi)(i), \quad i \geq 0 .
$$

Since equation (6) is Volterra type, we have

$$
f_{k} \circ\left(f_{i-1}, f_{i-2}, \ldots, f_{i-s}\right)=f_{k+i}, \quad k \geq-s, \quad i \geq 0 .
$$

In the following results necessary and sufficient conditions are given to establish whether or not every solution of equation (6) determined by initial values from a subset of $D_{a d}$ is periodic with period $p$.

First, $p \geq s$ is supposed.

Theorem 5 Consider equation (6), and let $p \geq s$ be an integer.

(a) If (6) is globally p-periodic on the set $A \subset D_{a d}$, then

$$
f_{p-s}\left(y_{1}, y_{2}, \ldots, y_{s}\right)=y_{s}
$$

for all $\left(y_{s}, y_{s-1}, \ldots, y_{1}\right) \in A$.

(b) If the set $A \subset D_{h}$ is invariant in the sence that $\left(y_{s}, \ldots, y_{1}\right) \in A$ implies

$$
\left(y_{s-1}, \ldots, y_{1}, h\left(y_{1}, \ldots, y_{s}\right)\right) \in A,
$$

then $A \subset D_{a d}$ and the converse of the statement (a) is also valid.

We stress the case $p=s$.

Corollary 6 Consider equation (6). If (6) is globally s-periodic on the set $A \subset D_{a d}$, then

$$
h\left(y_{1}, y_{2}, \ldots, y_{s}\right)=y_{s}
$$

for all $\left(y_{s}, y_{s-1}, \ldots, y_{1}\right) \in A$.

Remark 7 Assume $A=D_{a d}$. Obviously, $D_{a d}$ is an invariant set, and hence Theorem 5 can be written in a more compact form: equation (6) is globally p-periodic if and only if (10) holds for all $\left(y_{s}, y_{s-1}, \ldots, y_{1}\right) \in D_{a d}$.

Now we consider the case $1 \leq p<s$. If $a \in \mathbb{R}$, then $[a]$ denotes the greatest integer that does not exceed $a$.

Theorem 8 Consider equation (6), let $1 \leq p<s$ be an integer, and let $u:=$ $\left[\frac{s}{p}\right]$ and $v:=s-u p(0 \leq v \leq p-1)$. Define

$D_{a d, p}:=\left\{\varphi=(\varphi(-s), \ldots, \varphi(-1)) \in D_{a d} \mid \varphi(i)=\varphi(j)\right.$ if $\left.i \equiv j(\bmod p)\right\}$.

(a) If (6) is globally p-periodic on the set $A \subset D_{a d, p}$, then 
(a $a_{1}$ in case $v=0$

$$
h\left(y_{1}, y_{2}, \ldots, y_{p}, \ldots, y_{1}, y_{2}, \ldots, y_{p}\right)=y_{p}
$$

for all $\left(y_{p}, y_{p-1}, \ldots, y_{1}, \ldots, y_{p}, y_{p-1}, \ldots, y_{1}\right) \in A$,

$\left(a_{2}\right)$ in case $0<v \leq p-1$

$$
h\left(y_{1}, y_{2}, \ldots, y_{p}, \ldots, y_{1}, y_{2}, \ldots, y_{p}, y_{1}, \ldots, y_{v}\right)=y_{p}
$$

for all $\left(y_{v}, \ldots, y_{1}, y_{p}, y_{p-1}, \ldots, y_{1}, \ldots, y_{p}, y_{p-1}, \ldots, y_{1}\right) \in A$.

(b) Suppose the set

$$
A \subset\left\{\left(y_{s}, \ldots, y_{1}\right) \in X^{s} \mid\left(y_{1}, \ldots, y_{s}\right) \in D_{h}, \quad y_{i}=y_{j} \text { if } i \equiv j(\bmod p)\right\}
$$

is invariant in the sence that $\left(y_{s}, \ldots, y_{1}\right) \in A$ implies

$$
\begin{gathered}
\left(y_{s-1}, \ldots, y_{1}, y_{p}\right) \in A \\
\left(y_{s-2}, \ldots, y_{1}, y_{p}, y_{p-1}\right) \in A \\
\vdots \\
\left(y_{s-p+1}, \ldots, y_{1}, y_{p}, y_{p-1}, \ldots, y_{2}\right) \in A
\end{gathered}
$$

and either $\left(a_{1}\right)$ or $\left(a_{2}\right)$ holds. Then $A \subset D_{a d, p}$, and (6) is globally p-periodic on $A$.

As an illustration to Theorem 5, we consider the system of the sth order inhomogeneous linear difference equations

$$
x(n)=\sum_{i=1}^{s} A_{i} x(n-i)+b, \quad n \geq 0,
$$

where $s \geq 1$ is a given integer, $X$ is a real vector space, $A_{i} \in \mathcal{L}(X)$ for every $1 \leq i \leq s$, and $b \in X$.

$\mathcal{L}(X)$ means the real vector space of linear (additive and homogeneous) mappings from $X$ into $X, I$ is the identity mapping on $X$ and $O$ is the zero element in $\mathcal{L}(X)$.

The system of the $s$ th order homogeneous linear difference equations associated (11) is

$$
x(n)=\sum_{i=1}^{s} A_{i} x(n-i), \quad n \geq 0 .
$$

It is clear that (11) is a special case of (6), where $h: X^{s} \rightarrow X$,

$$
h\left(y_{1}, \ldots, y_{s}\right)=\sum_{i=1}^{s} A_{i} y_{i}+b .
$$

In this case the function $f_{i}(i \geq 0)$ in (8) has the form

$$
f_{i}\left(y_{1}, y_{2}, \ldots, y_{s}\right)=\sum_{j=1}^{s} A_{j}^{(i)} y_{i}+B^{(i)} b, \quad\left(y_{s}, \ldots, y_{1}\right) \in D_{a d}=X^{s},
$$


where $A_{j}^{(i)} \in \mathcal{L}(X)$ for every $1 \leq j \leq s$, and $B^{(i)} \in \mathcal{L}(X)$. By using the definition of $f_{i}$, an easy calculation shows that

$$
A_{j}^{(0)}=A_{j}, \quad 1 \leq j \leq s, \quad B^{(0)}=I,
$$

and for $1 \leq i<s$

$$
\begin{gathered}
A_{j}^{(i)}=\sum_{k=1}^{i} A_{k} A_{j}^{(i-k)}+A_{j+i}, \quad 1 \leq j \leq s-i, \\
A_{j}^{(i)}=\sum_{k=1}^{i} A_{k} A_{j}^{(i-k)}, \quad s-i+1 \leq j \leq s, \\
B^{(i)}=\sum_{k=1}^{i} A_{k} B^{(i-k)}+I,
\end{gathered}
$$

and for $i \geq s$

$$
A_{j}^{(i)}=\sum_{k=1}^{s} A_{k} A_{j}^{(i-k)}, \quad 1 \leq j \leq s, \quad B^{(i)}=\sum_{k=1}^{s} A_{k} B^{(i-k)}+I .
$$

The following result establishes the connection of (13) with Theorem 5 .

Corollary 9 Consider equation (11), and let $p \geq s$ be an integer.

(a) (11) is globally p-periodic if and only if

$$
A_{j}^{(p-s)}=O, \quad 1 \leq j \leq s-1, \quad A_{s}^{(p-s)}=I, \quad B^{(p-s)} b=0 .
$$

(b) (11) is globally p-periodic if and only if (12) is globally p-periodic and

$$
B^{(p-s)} b=0 \text {. }
$$

Now, we describe explicitly the cases $p=s$ and $p=s+1$.

Corollary 10 Consider equation (11).

(a) (11) is globally s-periodic if and only if

$$
A_{1}=\ldots=A_{s-1}=O, \quad A_{s}=I, \quad b=0 .
$$

(b) (11) is globally $(s+1)$-periodic if and only if

$$
A_{1}^{s+1}=(-1)^{s+1} I, \quad A_{i}=(-1)^{i+1} A_{1}^{i}, \quad 2 \leq i \leq s, \quad\left(A_{1}+I\right) b=0 .
$$

If $X$ is finite dimensional, another approach can also be applied.

The zero matrix and the identity matrix in $\mathbb{R}^{d \times d}$ are denoted by $O_{d}$ and $I_{d}$, respectively. 
Remark 11 Suppose $X=\mathbb{R}^{d}, A_{i} \in \mathbb{R}^{d \times d}$ for every $1 \leq i \leq s$, and $b \in \mathbb{R}^{d}$. Let $p \geq s$ be an integer. It follows from Theorem 1.2 in [19] that equation (12) is is globally p-periodic if and only if the rank of the matrix

$$
\left(\begin{array}{ccccc}
B_{p}-I_{d} & B_{p-1} & \ldots & B_{2} & B_{1} \\
B_{1} & B_{p}-I_{d} & \ldots & B_{3} & B_{2} \\
\vdots & \vdots & \ddots & \vdots & \vdots \\
B_{p-1} & B_{p-2} & \ldots & B_{1} & B_{p}-I_{d}
\end{array}\right)
$$

with entries

$$
B_{i}:=A_{i}, \quad 1 \leq i \leq s \text { and } B_{i}:=O_{d}, \quad s+1 \leq i \leq p .
$$

is equal to $d(p-s)$.

\subsection{A necessary condition for global periodicity via the linearized equation}

We study equation (3)

$$
x(n)=h(x(n-1), \ldots, x(n-s)), \quad n \geq 0,
$$

in that case when $s \geq 1$ is a given integer, $X$ is a real normed space, and $h$ : $D_{h}\left(\subset X^{s}\right) \rightarrow X$.

We say that the point $a \in X$ is an equilibrium of equation (14), if $h(a, \ldots, a)=$ $a$. If $a \in X$ is an equilibrium of equation (14) and $h$ is (Fréchet) differentiable at $\varphi_{e}=(a, \ldots, a) \in X^{s}$, then the $s$ th order linear difference equation

$$
x(n)=a+\sum_{i=1}^{s} d_{i} h\left(\varphi_{e}\right)(x(n-i)-a), \quad n \geq 0
$$

is called the linearized equation of (14) around $a$. Here $d_{i} h(1 \leq i \leq s)$ means the partial differential of $h$ with respect to the $i$ th coordinate. $d_{i} h\left(\varphi_{e}\right)(1 \leq i \leq s)$ is a continuous linear mapping from $X$ into $X$. The homogeneous linear difference equation associated (15) is

$$
x(n)=\sum_{i=1}^{s} d_{i} h\left(\varphi_{e}\right) x(n-i), \quad n \geq 0 .
$$

Theorem 12 Consider equation (14), let $a \in X$ be an equilibrium point of (14), and let $h$ be differentiable at $\varphi_{e}=(a, \ldots, a) \in X^{s}$. If there is a neigbourhood $U \subset D_{a d}$ of $\varphi_{e}$ such that (14) is globally p-periodic on $U$ with a fixed $p \geq s$, then equation (16) is globally p-periodic too.

The previous result is an essential generalization of former results in which only the finite dimensional case is considered. $X=\mathbb{R}^{k}$ for some $k=1,2, \ldots$ in Theorem 3.2 from [22], and the analyticity of $h$ in a neigbourhood of $\varphi_{e}$ is 
supposed. $h$ is a function from $I^{s}$ into $I$, where $I \subset \mathbb{R}$ is an interval, in Corollary 2 from [28], and it is assumed that $h$ is continuously differentiable. The proofs of these results are based on the utilization of the Jacobian matrix which does not exist if $X$ is not finite dimensional.

Remark 13 Let $X$ be a real normed space, and let $p \geq s$ be an integer. Consider the higher order linear difference equation

$$
x(n)=\sum_{i=1}^{s} A_{i} x(n-i)+b, \quad n \geq 0,
$$

where $s \geq 1$ is a given integer, $b \in X$, and $A_{i}$ is a continuous linear map from $X$ into itself for every $1 \leq i \leq s$. The homogeneous linear difference equation associated (17) is

$$
x(n)=\sum_{i=1}^{s} A_{i} x(n-i), \quad n \geq 0 .
$$

It is well known that the general solution of the nonhomogeneous equation (17) can be written as the sum of a particular solution of equation (17) and the general solution of equation (18). It follows from this

(a) If (17) is globally p-periodic, then (18) is globally p-periodic too.

(b) If (17) has an equilibrium point, then the converse of (a) is also true.

In the one-dimensional case the existence of an equilibrium point is necessary to the global periodicity of equation (17). Remark 13 (a) can be reversed.

Theorem 14 Consider equation (17) in the scalar case that is $A_{i} \in \mathbb{R}(1 \leq i \leq s)$ and $b \in \mathbb{R}$. Suppose $s \geq 1$ is a given integer.

(a) If (17) has not got an equilibrium point, then there exists an unbounded solution.

(b) Let $p \geq s$ be an integer. Then (17) is globally p-periodic if and only if (18) is globally p-periodic and (17) has an equilibrium point.

As applications of these results some difference equations will be studied.

First, we consider equation

$$
x(n)=\alpha|x(n-1)|+\beta|x(n-2)|+\gamma, \quad n \geq 0,
$$

where $\alpha, \beta$ and $\gamma$ are real numbers, and $\gamma \neq 0$.

Since $\gamma \neq 0,0$ is not an equilibrium point of equation (19). It is easy to check that (19) has a positive equilibrium point

$$
a_{p}:=\frac{\gamma}{1-(\alpha+\beta)}
$$

if either $\gamma>0$ and $\alpha+\beta<1$, or $\gamma<0$ and $\alpha+\beta>1$, and it has a negative equilibrium point

$$
a_{n}:=\frac{\gamma}{1+\alpha+\beta},
$$


if either $\gamma>0$ and $\alpha+\beta<-1$, or $\gamma<0$ and $\alpha+\beta>-1$.

It does not exist any equilibrium point of equation (19) if and only if either $\alpha+\beta=1$ and $\gamma>0$, or $\alpha+\beta=-1$ and $\gamma<0$.

We now obtain the following result.

Theorem 15 Consider equation (19). Let $p \geq 2$ be an integer.

(a) There is a neigbourhood $U \subset \mathbb{R}^{2}$ of $\left(a_{p}, a_{p}\right)$ such that (19) is globally p-periodic on $U$ if and only if

$$
\gamma>0, \quad \beta=-1 \quad \text { and } \quad \alpha=2 \cos \left(\frac{2 \pi}{p} k\right), \quad 1 \leq k<\frac{p}{2} \quad \text { is an integer. }
$$

(b) There is a neigbourhood $U \subset \mathbb{R}^{2}$ of $\left(a_{n}, a_{n}\right)$ such that (19) is globally p-periodic on $U$ if and only if

$$
\gamma<0, \quad \beta=1 \quad \text { and } \quad \alpha=-2 \cos \left(\frac{2 \pi}{p} k\right), \quad 1 \leq k<\frac{p}{2} \quad \text { is an integer. }
$$

(20) and (21) show that equation (19) can have a positive equilibrium point and a negative equilibrium point together. If there are two equilibrium points, then by $(22)$ and (23), at most one of $\left(a_{p}, a_{p}\right)$ and $\left(a_{n}, a_{n}\right)$ can have a neighbourhood $U$ such that every solution of (19) determined by an initial value from $U$ is periodic with the same period. But it can be occurred that one of the previous points has a neighbourhood with periodicity properties, while the other does not. This is illustrated by the next example.

Example 16 Equation

$$
x(n)=-|x(n-1)|-|x(n-2)|+3, \quad n \geq 0
$$

has two equilibrium points, namely $a_{p}=1$ and $a_{n}=-3$.

By Theorem 15 (a), there is a neigbourhood $U \subset \mathbb{R}^{2}$ of $(1,1)$ such that (24) is globally 3-periodic on $U$. For example, $U=] \frac{1}{2}, \frac{3}{2}[\times] \frac{1}{2}, \frac{3}{2}[$ can be choosen (see (96)).

But Theorem 15 (b) implies that there is not a neigbourhood $V \subset \mathbb{R}^{2}$ of $(-3,-3)$ such that every solution of (24) determined by an initial value from $V$ is periodic with the same period. For example, the solution of (19) determined by the initial value $(-4,-4)$ is

$$
x(-2)=-4, \quad x(n)=-4-F_{n+1}, \quad n \geq-1,
$$

where $\left(F_{n}\right)_{n=0}^{\infty}$ is the Fibonacci sequence, and thus it is not bounded.

Now, as a second application, we consider equation

$$
x(n)=\alpha|x(n-1)|+\beta x(n-2)+\gamma, \quad n \geq 0,
$$

where $\alpha, \beta$ and $\gamma$ are real numbers, and $\gamma \neq 0$. If $\gamma=0$, then (25) is called the Lozi equation, whose global periodicity was investigated in [1]. 
It follows from $\gamma \neq 0$ that 0 is not an equilibrium point of equation (25). It is not hard to check that equation (25) has a positive equilibrium point

$$
b_{p}:=\frac{\gamma}{1-(\alpha+\beta)},
$$

if either $\gamma>0$ and $\alpha+\beta<1$, or $\gamma<0$ and $\alpha+\beta>1$, and it has a negative equilibrium point

$$
b_{n}:=\frac{\gamma}{1-(\beta-\alpha)},
$$

if either $\gamma>0$ and $\beta-\alpha>1$, or $\gamma<0$ and $\beta-\alpha<1$.

It can be seen that equation (25) has not got any equilibrium points if and only if $\gamma \alpha>0$ and either $\beta+\alpha=1$ or $\beta-\alpha=1$.

We now make the following claim:

Theorem 17 Consider equation (25), and suppose there exists an equilibrium point of (25). Let $p \geq 2$ be an integer.

(a) There is a neigbourhood $U \subset \mathbb{R}^{2}$ of $\left(b_{p}, b_{p}\right)$ such that (25) is globally p-periodic on $U$ if and only if

$$
\gamma>0, \quad \beta=-1 \quad \text { and } \quad \alpha=2 \cos \left(\frac{2 \pi}{p} k\right), \quad 1 \leq k<\frac{p}{2} \quad \text { is an integer. }
$$

(b) There is a neigbourhood $U \subset \mathbb{R}^{2}$ of $\left(b_{n}, b_{n}\right)$ such that (25) is globally p-periodic on $U$ if and only if

$$
\gamma<0, \quad \beta=-1 \quad \text { and } \quad \alpha=-2 \cos \left(\frac{2 \pi}{p} k\right), \quad 1 \leq k<\frac{p}{2} \quad \text { is an integer. }
$$

The next example corresponds to Example 16.

Example 18 Consider the following special case of equation (25)

$$
x(n)=-|x(n-1)|-x(n-2)+3, \quad n \geq 0,
$$

which has only the equilibrium point 1. Theorem 17 (a) implies that there is a neigbourhood $U \subset \mathbb{R}^{2}$ of $(1,1)$ such that (28) globally 3-periodic on $U$. It is not true that every solution of (28) is 3-periodic: for example, the solution determined by the initial value $(-2,-2)$ has prime period 19.

Remark 19 Consider equation (25), and suppose there exists an equilibrium point of (25). Let $p \geq 2$ be an integer. It would be interesting to prove or disprove: If either (26) or (27) holds, then equation (25) is globally p-periodic.

Before we advert the next difference equation, we need the following notations: for a compact interval $J \subset \mathbb{R}, C(J, \mathbb{R})$ will denote the Banach space of all real-valued continuous functions defined on $J$ with the norm

$$
\|f\|_{\infty}:=\max _{t \in J}|f(x)| ;
$$


for $c \in \mathbb{R}, c_{J}$ means the identically $c$ constant function from $C(J, \mathbb{R})$.

Now consider equation

$$
x(n)=h(x(n-1)), \quad n \geq 0,
$$

where $J$ is a fixed compact interval in $\mathbb{R}$ with positive length, $f: J \times \mathbb{R} \rightarrow \mathbb{R}$ is a fixed continuous function whose partial differential with respect to the second variable $d_{2} f: J \times \mathbb{R} \rightarrow \mathbb{R}$ is continuous and $f(t, 0)=0$ for every $t \in J$, and the function $h: C(J, \mathbb{R}) \rightarrow C(J, \mathbb{R})$ is the Nemitcky operator given by

$$
h(\omega)(t):=f(t, \omega(t)), \quad t \in J .
$$

In this case equation (29) can be written in the form

$$
x(n)(t)=f(t, x(n-1)(t)), \quad t \in J, \quad n \geq 0 .
$$

Theorem 20 Consider equation (29), and let $p \geq 1$ be an integer. (29) is globally p-periodic only if either

$$
d_{2} f(t, 0)=1, \quad t \in J,
$$

or $p$ is even and

$$
d_{2} f(t, 0)=-1, \quad t \in J .
$$

Proof. The condition $f(t, 0)=0$ for every $t \in J$ shows that $0_{J}$ is an equilibrium point of (29). It can be proved (see [14]) that the other conditions on $f$ guarantee the differentiability of $h$ on $C(J, \mathbb{R})$ and for each $\omega \in C(J, \mathbb{R})$ and all $\eta \in C(J, \mathbb{R})$

$$
(d h(\omega)(\eta))(t)=d_{2} f(t, \omega(t)) \eta(t), \quad t \in J .
$$

It therefore follows that the linearized equation of $(29)$ around $0_{J}$ is

$$
x(n)(t)=d_{2} f(t, 0) x(n-1)(t), \quad t \in J, \quad n \geq 0 .
$$

By applying Theorem 12, we have: if equation (29) is globally p-periodic, then equation (32) is also globally $p$-periodic. But the global $p$-periodicity of (32) is equivalent with

$$
d_{2} f(t, 0)^{p} \varphi(t)=\varphi(t), \quad t \in J
$$

for every $\varphi \in C(J, \mathbb{R})$. This gives

$$
d_{2} f(t, 0)^{p}=1, \quad t \in J .
$$

Hence either (30) is satisfied, or $p$ is even and (31) holds, which completes the proof.

We close this section with a lemma which will be useful in the proof of Theorem 15 . 
Lemma 21 Consider the difference equation

$$
x(n)=A_{1} x(n-1)+A_{2} x(n-2), \quad n \geq 0,
$$

where $A_{1}, A_{2} \in \mathbb{R}$.

(a) (33) is globally 2-periodic if and only if

$$
A_{2}=1 \text { and } A_{1}=0 .
$$

(b) Let $p \geq 3$ be an even integer. (33) is globally p-periodic if and only if either (34) holds or $A_{2}=-1$ and there is an integer $1 \leq k<\frac{p}{2}$ such that

$$
A_{1}=2 \cos \left(\frac{2 \pi}{p} k\right) .
$$

(c) Let $p \geq 3$ be an odd integer. (33) is globally p-periodic if and only if $A_{2}=-1$ and there is an integer $1 \leq k<\frac{p}{2}$ such that (35) is satisfied.

\section{$3 \quad$ Further results}

We need some preparatory results, which are interesting in their own rights.

\subsection{Circulant system of equations and circulant matrices}

Suppose $X$ is a nonempty set, and $s$ and $p$ are positive integers. If $y=$ $\left(y_{p}, \ldots, y_{1}\right) \in X^{p}$ is a row vector, then the related column vector is denoted by $y^{T}=\left(y_{p}, \ldots, y_{1}\right)^{T}$.

The following concepts enable us to conceive the results of the next section as clear as possible.

Definition 22 The shift operator $S: X^{p} \rightarrow X^{p}$ is defined by

$$
S y=S\left(y_{p}, \ldots, y_{1}\right):=\left(y_{1}, y_{p}, \ldots, y_{2}\right),
$$

and its iterations $S^{k}$ are defined by

$$
S^{k} y:=S\left(S^{k-1} y\right), \quad k \geq 1,
$$

where $S^{0} y:=y$. For $k \geq 1, S^{-k}$ denotes the inverse operator of $S^{k}$ (obviously $\left.S^{-k}=\left(S^{-1}\right)^{k}\right)$.

Definition 23 Let $g_{i}: D_{g_{i}}\left(\subset X^{s}\right) \rightarrow X(1 \leq i \leq p)$ be given mappings. The system of equations

$$
\left.\begin{array}{c}
y_{p}=g_{1}\left((S y)_{1}^{T}, \ldots,\left(S^{s} y\right)_{1}^{T}\right) \\
\vdots \\
y_{1}=g_{p}\left((S y)_{p}^{T}, \ldots,\left(S^{s} y\right)_{p}^{T}\right)
\end{array}\right\}
$$

is called column-wise circulant system, where $y=\left(y_{p}, \ldots, y_{1}\right) \in X^{p}$ is the unknown row vector. 
Column-wise circulant systems are illustrated by the next examples.

Example 24 We consider three cases: $p<s, p=s$ and $p>s$. $p$ will be fixed: $p=4$, and therefore

$$
\begin{gathered}
y=\left(y_{4}, y_{3}, y_{2}, y_{1}\right), \quad S y=\left(y_{1}, y_{4}, y_{3}, y_{2}\right), \quad S^{2} y=\left(y_{2}, y_{1}, y_{4}, y_{3}\right) \\
S^{3} y=\left(y_{3}, y_{2}, y_{1}, y_{4}\right), \quad S^{4} y=\left(y_{4}, y_{3}, y_{2}, y_{1}\right)=y .
\end{gathered}
$$

In the first example $s=6$, while in the next one $s=4$ :

$$
\left.\left.\begin{array}{l}
y_{4}=g_{1}\left(y_{1}, y_{2}, y_{3}, y_{4}, y_{1}, y_{2}\right) \\
y_{3}=g_{2}\left(y_{4}, y_{1}, y_{2}, y_{3}, y_{4}, y_{1}\right) \\
y_{2}=g_{3}\left(y_{3}, y_{4}, y_{1}, y_{2}, y_{3}, y_{4}\right) \\
y_{1}=g_{4}\left(y_{2}, y_{3}, y_{4}, y_{1}, y_{2}, y_{3}\right)
\end{array}\right\}, \begin{array}{l}
y_{4}=g_{1}\left(y_{1}, y_{2}, y_{3}, y_{4}\right) \\
y_{3}=g_{2}\left(y_{4}, y_{1}, y_{2}, y_{3}\right) \\
y_{2}=g_{3}\left(y_{3}, y_{4}, y_{1}, y_{2}\right) \\
y_{1}=g_{4}\left(y_{2}, y_{3}, y_{4}, y_{1}\right)
\end{array}\right\} .
$$

Finally, $s=3$ :

$$
\left.\begin{array}{l}
y_{4}=g_{1}\left(y_{1}, y_{2}, y_{3}\right) \\
y_{3}=g_{2}\left(y_{4}, y_{1}, y_{2}\right) \\
y_{2}=g_{3}\left(y_{3}, y_{4}, y_{1}\right) \\
y_{1}=g_{4}\left(y_{2}, y_{3}, y_{4}\right)
\end{array}\right\} \text {. }
$$

Remark 25 The above examples clearly show that a system of equations is column-wise circulant if each column of unknowns (after the first) has the elements of the previous column of unknowns shifted cyclically one place down.

The system of linear equations

$$
\left.\begin{array}{c}
y_{p}=a_{1}(S y)_{1}^{T}+\ldots+a_{s}\left(S^{s} y\right)_{1}^{T}+b_{p} \\
\vdots \\
y_{1}=a_{1}(S y)_{p}^{T}+\ldots+a_{s}\left(S^{s} y\right)_{p}^{T}+b_{1}
\end{array}\right\}
$$

is a column-wise circulant linear system. If $p=s$, then this system can be written as

$$
\begin{gathered}
y_{s}=a_{1} y_{1}+a_{2} y_{2}+\ldots+a_{s} y_{s}+b_{s} \\
y_{s-1}=a_{s} y_{s}+a_{1} y_{1}+\ldots+a_{s-1} y_{s-1}+b_{s-1} \\
\vdots \\
y_{1}=a_{2} y_{2}+a_{3} y_{3}+\ldots+a_{1} y_{1}+b_{1}
\end{gathered}
$$

which has the matrix form

$$
\left(\begin{array}{c}
y_{s} \\
y_{s-1} \\
\vdots \\
y_{1}
\end{array}\right)=\left(\begin{array}{cccc}
a_{s} & a_{s-1} & \ldots & a_{1} \\
a_{1} & a_{s} & \ldots & a_{2} \\
\vdots & \vdots & \ddots & \vdots \\
a_{s-1} & a_{s-2} & \ldots & a_{s}
\end{array}\right)\left(\begin{array}{c}
y_{s} \\
y_{s-1} \\
\vdots \\
y_{1}
\end{array}\right)+\left(\begin{array}{c}
b_{s} \\
b_{s-1} \\
\vdots \\
b_{1}
\end{array}\right) .
$$

The matrix $A$ of the above equation is a circulant matrix associated to the first row

$$
A=\left(a_{s}, \ldots, a_{1}\right)
$$

in which each row (after the first) has the elements of the previous row shifted cyclically one place right. 


\subsection{Existence of periodic solutions of higher order differ- ence systems}

In the first theorem a method is given to determine the existence of $s$-periodic solutions of the equation (1).

Theorem 26 Consider equation (1).

(a) If $\varphi=(\varphi(-s), \ldots, \varphi(-1)) \in D_{a d}$ is an s-periodic initial vector of (1), then $\varphi$ is a solution $\left(y_{i}=\varphi(-i), 1 \leq i \leq s\right)$ of the column circulant system of equations

$$
\left.\begin{array}{c}
y_{s}=h_{k s-s}\left(y_{1}, y_{2}, \ldots, y_{s-1}, y_{s}\right) \\
y_{s-1}=h_{k s-s+1}\left(y_{s}, y_{1}, \ldots, y_{s-2}, y_{s-1}\right) \\
\vdots \\
y_{1}=h_{k s-1}\left(y_{2}, y_{3}, \ldots, y_{s}, y_{1}\right)
\end{array}\right\}
$$

for all $k \geq 1$.

(b) If $\varphi:=\left(y_{s}, \ldots, y_{1}\right) \in X^{s}$ is a solution of (36) for all $k \geq 1$, then $\varphi \in D_{a d}$ and $\varphi$ is an s-periodic initial vector of (1).

Next, we study the existence of $p$-periodic solutions of the equation (1), when $p \neq s$. We begin with the case $p>s$.

Theorem 27 Consider equation (1), and let $p>s$ be an integer. then

(a) If $\varphi=(\varphi(-s), \ldots, \varphi(-1)) \in D_{a d}$ is a p-periodic initial vector of (1),

$$
\psi:=(x(\varphi)(0), \ldots, x(\varphi)(p-s-1), \varphi(-s), \ldots, \varphi(-1)) \in X^{p}
$$

is a solution of the column circulant system of equations

$$
\left.\begin{array}{c}
y_{p}=h_{k p-p}\left(y_{1}, y_{2}, \ldots, y_{s}\right) \\
y_{p-1}=h_{k p-p+1}\left(y_{p}, y_{1}, \ldots, y_{s-1}\right) \\
\vdots \\
y_{1}=h_{k p-1}\left(y_{2}, y_{3}, \ldots, y_{s+1}\right)
\end{array}\right\}
$$

for all $k \geq 1$.

(b) If $\psi:=\left(y_{p}, \ldots, y_{1}\right) \in X^{p}$ is a solution of (37) for all $k \geq 1$, then $\varphi=\left(y_{s}, \ldots, y_{1}\right) \in D_{a d}$ and $\varphi$ is a p-periodic initial vector of (1).

We turn now to the case $1 \leq p<s$.

Theorem 28 Consider equation (1), let $1 \leq p<s$ be an integer, and let $u:=\left[\frac{s}{p}\right]$ and $v:=s-u p(0 \leq v \leq p-1)$.

(a) If $\varphi=(\varphi(-s), \ldots, \varphi(-1)) \in D_{a d}$ is a p-periodic initial vector of (1), then $\psi:=(\varphi(-p), \ldots, \varphi(-1)) \in X^{p}$ is a solution $\left(y_{i}=\varphi(-i), 1 \leq i \leq p\right)$ one of the column circulant systems of equations for all $k \geq 1$ : 
(a $a_{1}$ in case $v=0$

$$
\left.\begin{array}{c}
y_{p}=h_{k p-p}\left(y_{1}, y_{2}, \ldots, y_{p}, \ldots, y_{1}, y_{2}, \ldots, y_{p}\right) \\
y_{p-1}=h_{k p-p+1}\left(y_{p}, y_{1}, \ldots, y_{p-1}, \ldots, y_{p}, y_{1}, \ldots, y_{p-1}\right) \\
\vdots \\
y_{1}=h_{k p-1}\left(y_{2}, y_{3}, \ldots, y_{1}, \ldots, y_{2}, y_{3}, \ldots, y_{1}\right)
\end{array}\right\},
$$

$\left(a_{2}\right)$ in case $0<v \leq p-1$

$$
\left.\begin{array}{c}
y_{p}=h_{k p-p}\left(y_{1}, y_{2}, \ldots, y_{p}, \ldots, y_{1}, y_{2}, \ldots, y_{p}, y_{1}, \ldots, y_{v}\right) \\
y_{p-1}=h_{k p-p+1}\left(y_{p}, y_{1}, \ldots, y_{p-1}, \ldots, y_{p}, y_{1}, \ldots, y_{p-1}, y_{p}, \ldots y_{v-1}\right) \\
\vdots \\
y_{1}=h_{k p-1}\left(y_{2}, y_{3}, \ldots, y_{1}, \ldots, y_{2}, y_{3}, \ldots, y_{1}, y_{2}, \ldots, y_{v+1}\right)
\end{array}\right\} .
$$

(b) If $\psi:=\left(y_{p}, \ldots, y_{1}\right) \in X^{p}$ is a solution of either (38) or (39) (depending on $v$ ) for all $k \geq 1$, then $\varphi=(\varphi(-s), \ldots, \varphi(-1))$ defined by $\varphi(i):=y_{j}$ if $-i \equiv j$ $(\bmod p)(-s \leq i \leq-1,1 \leq j \leq p)$ belongs to $D_{a d}$ and $\varphi$ is a p-periodic initial vector of $(1$.

To illustrate the above results we consider the system of the sth order inhomogeneous linear difference equations

$$
x(n)=\sum_{i=1}^{s} A_{i}(n) x(n-i)+f(n), \quad n \geq 0,
$$

where $s \geq 1$ is a given integer, $A_{i}(n) \in \mathbb{R}^{d \times d}$ for every $1 \leq i \leq s$ and $n \geq 0$, and $f(n) \in \mathbb{R}^{d}$ for every $n \geq 0$.

For every positive integer $p V_{p}$ means the $p d$-dimensional real vector space of block column vectors with entries in $\mathbb{R}^{d}$. The zero matrix in $\mathbb{R}^{d \times d}$ is denoted by $O$.

Theorem 26 gives a surprisingly simple way to determine the existence of $s$-periodic solutions of equation (40).

Theorem 29 Consider equation (40). Let $\varphi=(\varphi(-s), \ldots, \varphi(-1))^{T} \in V_{s}$ be a given initial vector. The solution $x(\varphi)$ of (40) and (2) is s-periodic if and only if

$$
\mathcal{B}_{s}^{(k)} \varphi+\mathcal{F}_{s}^{(k)}=\varphi
$$

for every $k \geq 1$, where

$$
\mathcal{B}_{s}^{(k)}:=\left(\begin{array}{cccc}
A_{s}(k s-s) & A_{s-1}(k s-s) & \ldots & A_{1}(k s-s) \\
A_{1}(k s-s+1) & A_{s}(k s-s+1) & \ldots & A_{2}(k s-s+1) \\
\vdots & \vdots & \ddots & \vdots \\
A_{s-1}(k s-1) & A_{s-2}(k s-1) & \ldots & A_{s}(k s-1)
\end{array}\right)
$$


Proof. It follows immediately from Theorem 26.

Now, we formulate the consequences of Theorem 28 and Theorem 27. We need the following notations.

Definition 30 Consider equation (40), and let $p$ be a positive integer.

(a) Suppose $p<s$, and let $u:=\left[\frac{s}{p}\right]$ and $v:=s-u p(0 \leq v \leq p-1)$.

If $v=0$, define

$$
B_{i}(n):=\sum_{j=0}^{u-1} A_{j p+i}(n), \quad 1 \leq i \leq p,
$$

while if $v \neq 0$, define

$$
B_{i}(n):=\left\{\begin{array}{c}
\sum_{j=0}^{u} A_{j p+i}(n), \quad 1 \leq i \leq v \\
\sum_{j=0}^{u-1} A_{j p+i}(n), \quad v+1 \leq i \leq p
\end{array} .\right.
$$

(b) If $p>s$, define

$$
B_{i}(n):=\left\{\begin{array}{l}
A_{i}(n), \quad 1 \leq i \leq s \\
O, \quad s+1 \leq i \leq p
\end{array} .\right.
$$

(c) For every positive integer $k$ define the block matrix

$$
\mathcal{B}_{p}^{(k)}=\left(\begin{array}{cccc}
B_{p}(k p-p) & B_{p-1}(k p-p) & \ldots & B_{1}(k p-p) \\
B_{1}(k p-p+1) & B_{p}(k p-p+1) & \ldots & B_{2}(k p-p+1) \\
\vdots & \vdots & \ddots & \vdots \\
B_{p-1}(k p-1) & B_{p-2}(k p-1) & \ldots & B_{p}(k p-1)
\end{array}\right),
$$

and the block vector

$$
\mathcal{F}_{p}^{(k)}:=(f(k p-p), \ldots, f(k p-1))^{T} .
$$

Theorem 31 Consider equation (40). Suppose $1 \leq p<s$ is an integer, and let $u:=\left[\frac{s}{p}\right]$ and $v:=s-u p(0 \leq v \leq p-1)$.

(a) If $\varphi=(\varphi(-s), \ldots, \varphi(-1))^{T} \in V_{s}$ is a p-periodic initial vector of (40), and $\psi:=(\varphi(-p), \ldots, \varphi(-1))^{T} \in V_{p}$, then

$$
\mathcal{B}_{p}^{(k)} \psi+\mathcal{F}_{p}^{(k)}=\psi
$$

for every $k \geq 1$, where $\mathcal{B}_{p}^{(k)}$ is the matrix with entries defined in Definition 30 (a).

Conversely, if $\psi=(\psi(-p), \ldots, \psi(-1))^{T} \in V_{p}$ such that (42) is satisfied for every $k \geq 1$, then $\varphi=(\varphi(-s), \ldots, \varphi(-1))^{T} \in V_{s}$, where $\varphi(i):=\psi(j)$ if $i \equiv j$ $(\bmod p)(-s \leq i \leq-1,-p \leq j \leq-1)$, is a p-periodic initial vector of $(40)$.

(b) (40) has a p-periodic solution if and only if there exists $a \psi \in V_{p}$ such that (42) is satisfied for every $k \geq 1$. 
Proof. Theorem 28 can be applied.

Theorem 32 Consider equation (40). Suppose $p>s$ is an integer.

(a) If $\varphi=(\varphi(-s), \ldots, \varphi(-1))^{T} \in V_{s}$ is a p-periodic initial vector of (40), and

$$
\psi:=(x(\varphi)(0), \ldots, x(\varphi)(p-s-1), \varphi(-s), \ldots, \varphi(-1))^{T} \in V_{p},
$$

then

$$
\mathcal{B}_{p}^{(k)} \psi+\mathcal{F}_{p}^{(k)}=\psi
$$

holds for every $k \geq 1$, where $\mathcal{B}_{p}^{(k)}$ is the matrix with entries defined in Definition 30 (b).

Conversely, if $\psi:=(\psi(-p), \ldots, \psi(-1))^{T} \in V_{p}$ such that (43) is satisfied for every $k \geq 1$, then $\varphi=(\psi(-s), \ldots, \psi(-1))^{T} \in V_{s}$ is a p-periodic initial vector of $(40)$.

(b) (40) has a p-periodic solution if and only if there exists a $\psi \in V_{p}$ such that (43) is satisfied for every $k \geq 1$.

Proof. This is an immediate consequence of Theorem 27.

Remark 33 (a) If $\mathcal{B}_{p}^{(k)}$ and $\mathcal{F}_{p}^{(k)}(k \geq 1)$ do not depend on $k$, then equations (41-43) can be handled easily, since we have to study only the equation

$$
\mathcal{B}_{p} \psi+\mathcal{F}_{p}=\psi
$$

where $\mathcal{B}_{p}:=\mathcal{B}_{p}^{(k)}, \mathcal{F}_{p}:=\mathcal{F}_{p}^{(k)}$.

(b) If equation (40) is homogeneous, then the results coincide with the corresponding results in [19].

\section{Applications}

\subsection{Global periodicity}

In the first example the conditions in Corollary 10 (b) will be studied in the case $s=d=2$.

Example 34 Consider the system of linear difference equations

$$
\left(\begin{array}{l}
x_{1}(n) \\
x_{2}(n)
\end{array}\right)=A_{1}\left(\begin{array}{c}
x_{1}(n-1) \\
x_{2}(n-1)
\end{array}\right)+A_{2}\left(\begin{array}{c}
x_{1}(n-2) \\
x_{2}(n-2)
\end{array}\right)+\left(\begin{array}{c}
b_{1} \\
b_{2}
\end{array}\right), \quad n \geq 0,
$$

where $A_{i} \in \mathbb{R}^{2 \times 2}$ for $i=1,2$ and $b=\left(b_{1}, b_{2}\right)^{T} \in \mathbb{R}^{2}$. Equation (44) is globally 3 -periodic if and only if either

$$
A_{1}=A_{2}=\left(\begin{array}{cc}
-1 & 0 \\
0 & -1
\end{array}\right)
$$


and $\left(b_{1}, b_{2}\right)^{T}$ is arbitrary, or

$$
A_{1}=\left(\begin{array}{cc}
1-d & \frac{-d^{2}+d-1}{c} \\
c & d
\end{array}\right), \quad c, d \in \mathbb{R}, \quad c \neq 0
$$

$A_{2}=A_{1}^{-1}$ and $\left(b_{1}, b_{2}\right)=(0,0)$.

Really, Corollary 10 (b) shows that

$$
A_{1}^{3}=\left(\begin{array}{ll}
a & b \\
c & d
\end{array}\right)^{3}=\left(\begin{array}{cc}
-1 & 0 \\
0 & -1
\end{array}\right)
$$

$A_{2}=A_{1}^{-1}$ and

$$
\left(A_{1}+I\right) b=0 .
$$

By solving the matrix equation (47), we obtain (45) and (46). Then (48) gives the conditions for $b$.

For example, the system of linear difference equations

$$
\left.\begin{array}{l}
x_{1}(n)=-x_{1}(n-1)-x_{2}(n-1)+2 x_{1}(n-2)+x_{2}(n-2) \\
x_{2}(n)=3 x_{1}(n-1)+2 x_{2}(n-1)-3 x_{1}(n-2)-x_{2}(n-2)
\end{array}\right\}, \quad n \geq 0
$$

is 3-globally periodic.

Next, we study some rational difference equations of the form

$$
x(n)=\frac{a_{0}+a_{1} x(n-1)+\ldots+a_{s} x(n-s)}{b_{0}+b_{1} x(n-1)+\ldots+b_{s} x(n-s)}, \quad n \geq 0,
$$

where $s \geq 3$ is a fixed positive integer and $a_{i}, b_{i} \in \mathbb{R}(0 \leq i \leq s)$. This equation is a special case of (6) with $h: D_{h}\left(\subset \mathbb{R}^{s}\right) \rightarrow \mathbb{R}$,

$$
h\left(y_{1}, \ldots, y_{s}\right)=\frac{a_{0}+a_{1} y_{1}+\ldots+a_{s} y_{s}}{b_{0}+b_{1} y_{1}+\ldots+b_{s} y_{s}}
$$

Let

$$
\begin{aligned}
A:=\left\{\left(y_{s}, \ldots, y_{1}\right) \in \mathbb{R}^{s} \mid y_{i}=y_{j} \text { if } i \equiv j(\bmod 2),\right. \\
\left.\left(y_{1}, \ldots, y_{s}\right), \quad\left(y_{s}, y_{1}, \ldots, y_{s-1}\right) \in D_{h}\right\} .
\end{aligned}
$$

We consider the case $s=2 t(t \geq 2)$, the other case $s=2 t+1(t \geq 1)$ can be handled similarly. Introduce the following numbers

$$
P_{e}:=\sum_{i=1}^{t} a_{2 i}, \quad P_{o}:=\sum_{i=1}^{t} a_{2 i-1}, \quad Q_{e}:=\sum_{i=1}^{t} b_{2 i}, \quad Q_{o}:=\sum_{i=1}^{t} b_{2 i-1} .
$$

(i) If $Q_{b} \neq 0$, then

$$
A=\left\{\left(z_{2}, z_{1}, \ldots, z_{2}, z_{1}\right) \in \mathbb{R}^{2 t} \mid\left(z_{2}, z_{1}\right) \in U\right\}
$$


where

$$
U:=\left\{\left(z_{2}, z_{1}\right) \in \mathbb{R}^{2} \mid z_{2} \neq \frac{1}{Q_{b}}\left(Q_{o} z_{1}+b_{0}\right), z_{1} \neq \frac{1}{Q_{b}}\left(Q_{o} z_{2}+b_{0}\right)\right\} .
$$

(ii) If $Q_{o} \neq 0$, then

$$
A=\left\{\left(z_{2}, z_{1}, \ldots, z_{2}, z_{1}\right) \in \mathbb{R}^{2 t} \mid\left(z_{2}, z_{1}\right) \in U\right\},
$$

where

$$
U:=\left\{\left(z_{2}, z_{1}\right) \in \mathbb{R}^{2} \mid z_{2} \neq \frac{1}{Q_{o}}\left(Q_{b} z_{1}+b_{0}\right), z_{1} \neq \frac{1}{Q_{o}}\left(Q_{b} z_{2}+b_{0}\right)\right\} .
$$

(iii) If $Q_{b}=Q_{o}=0$ and $b_{0} \neq 0$, then $\left(z_{2}, z_{1}\right) \in U=\mathbb{R}^{2}$.

(iv) If $Q_{b}=Q_{o}=0$ and $b_{0}=0$, then $A=\varnothing$.

Theorem 35 Suppose $s=2 t(t \geq 1)$, and suppose that one of the conditions (i)-(iii) are satisfied. Equation (49) is globally 2-periodic on $A$ if and only if

$$
a_{0}=0, \quad \sum_{i=1}^{t} a_{2 i-1}=0, \quad \sum_{i=1}^{t} a_{2 i}=b_{0}, \quad \sum_{i=1}^{t} b_{2 i-1}=\sum_{i=1}^{t} b_{2 i}=0 .
$$

Proof. Since $A$ is an invariant set, we can apply Theorem $8\left(\mathrm{a}_{1}\right)$ and (b): Equation (49) is globally 2-periodic on $A$ if and only if

$$
\frac{a_{0}+\left(\sum_{i=1}^{t} a_{2 i-1}\right) z_{1}+\left(\sum_{i=1}^{t} a_{2 i}\right) z_{2}}{b_{0}+\left(\sum_{i=1}^{t} b_{2 i-1}\right) z_{1}+\left(\sum_{i=1}^{t} b_{2 i}\right) z_{2}}=z_{2}, \quad\left(z_{2}, z_{1}\right) \in U
$$

and this is equivalent with (50).

Theorem 12 and Theorem 5 are illustrated by studying the difference equation

$$
x(n)=\frac{a_{0}+a_{1} x(n-1)+a_{2} x(n-2)}{b_{0}+b_{1} x(n-1)+b_{2} x(n-2)}, \quad n \geq 0,
$$

where $a_{i}, b_{i} \in \mathbb{R}(i=0,1,2)$ such that

$$
\operatorname{rank}\left(\begin{array}{ccc}
a_{0} & a_{1} & a_{2} \\
b_{0} & b_{1} & b_{2}
\end{array}\right)=2, \quad\left|a_{2}\right|+\left|b_{2}\right| \neq 0 \quad \text { and } \quad\left|b_{1}\right|+\left|b_{2}\right| \neq 0
$$

Assume $c \in \mathbb{R}$ is an equilibrium point of (51), or equivalently $c$ obeys the equation

$$
\left(b_{1}+b_{2}\right) c^{2}+\left(b_{0}-a_{1}-a_{2}\right) c-a_{0}=0
$$

and

$$
b_{0}+\left(b_{1}+b_{2}\right) c \neq 0 \text {. }
$$


A simple computation shows that the linearized equation of (51) around $c$ is the following

$$
y(n)=\frac{a_{1}-b_{1} c}{b_{0}+\left(b_{1}+b_{2}\right) c} y(n-1)+\frac{a_{2}-b_{2} c}{b_{0}+\left(b_{1}+b_{2}\right) c} y(n-2), \quad n \geq 0 .
$$

First, we give a lemma which will be used in the next result.

Lemma 36 Consider equation (51). If $c \in \mathbb{R}$ is an equilibrium point of (51), and (51) is globally $p$-periodic with $p \geq 2$, then there is a neighbourhood $U$ of $(c, c)$ such that $U \subset D_{a d}$.

Proof. $b_{0}+\left(b_{1}+b_{2}\right) c \neq 0$ implies that there is a neighbourhood $V$ of $(c, c)$ such that

$$
b_{0}+b_{1} y_{1}+b_{2} y_{2} \neq 0, \quad\left(y_{2}, y_{1}\right) \in V .
$$

Since equation (51) is globally $p$-periodic with $p \geq 2$, it is enough to prove that there is a neighbourhood $U$ of $(c, c)$ such that

$$
\begin{gathered}
\left(y_{2}, y_{1}\right)=\left(f_{-2}\left(y_{1}, y_{2}\right), f_{-1}\left(y_{1}, y_{2}\right)\right) \in V \\
\left(y_{1}, x\left(y_{2}, y_{1}\right)(0)\right)=\left(f_{-1}\left(y_{1}, y_{2}\right), f_{0}\left(y_{1}, y_{2}\right)\right) \in V \\
\vdots \\
\left(x\left(y_{2}, y_{1}\right)(p-3), x\left(y_{2}, y_{1}\right)(p-2)\right)=\left(f_{p-3}\left(y_{1}, y_{2}\right), f_{p-2}\left(y_{1}, y_{2}\right)\right) \in V
\end{gathered}
$$

for every $\left(y_{2}, y_{1}\right) \in U$. The result now comes the facts that the functions $\left(f_{i}, f_{i+1}\right)(-2 \leq i \leq p-3)$ are continuous and

$$
\left(f_{i}(c, c), f_{i+1}(c, c)\right)=(c, c), \quad-2 \leq i \leq p-3 .
$$

By applying Theorem 12 we have the following result.

Theorem 37 Consider equation (51).

(a) If there are two real equilibrium points $c_{1} \neq c_{2}$ of (51), then it is not globally 2-periodic.

(b) There are two real equilibrium points $c_{1} \neq c_{2}$ of (51), and (51) is globally p-periodic with $p \geq 3$ only if

$$
\begin{gathered}
b_{1}=0, \quad a_{2}=-b_{0}, \quad b_{2} \neq 0, \\
\left(2 b_{0}-a_{1}\right)^{2}+4 a_{0} b_{2}>0, \quad b_{0}\left(a_{1}-b_{0}\right) \neq a_{0} b_{2},
\end{gathered}
$$

and there are different integers $1 \leq k_{1}, k_{2}<\frac{p}{2}$ such that

$$
\begin{aligned}
& \frac{a_{1}}{a_{1}+\sqrt{\left(2 b_{0}-a_{1}\right)^{2}+4 a_{0} b_{2}}}=\cos \left(\frac{2 \pi}{p} k_{1}\right) \\
& \frac{a_{1}}{a_{1}-\sqrt{\left(2 b_{0}-a_{1}\right)^{2}+4 a_{0} b_{2}}}=\cos \left(\frac{2 \pi}{p} k_{2}\right) .
\end{aligned}
$$


Proof. If equation (51) has an equlibrium point $c \in \mathbb{R}$, and it is globally $p$ periodic with $p \geq 2$, then Lemma 36 and Theorem 12 imply that the linear equation (53) is globally $p$-periodic too.

(a) By the previous establishment and by Lemma 21 (a), equation (51) is globally 2-periodic only if the following relations are satisfied

$$
\begin{gathered}
a_{1}-b_{1} c_{i}=0, \quad i=1,2 \\
a_{2}=b_{0}+b_{1} c_{i}+2 b_{2} c_{i}, \quad i=1,2 .
\end{gathered}
$$

Since $c_{1} \neq c_{2},(57)$ shows that

$$
a_{1}=b_{1}=0
$$

Therefore (58) has the form

$$
a_{2}=b_{0}+2 b_{2} c_{i}, \quad i=1,2 .
$$

Thus by using $c_{1} \neq c_{2}$ again, we have

$$
b_{2}=0, \quad a_{2}=b_{0} .
$$

But $b_{1}=b_{2}=0$ contradicts to the third condition in (52) (and also the assumption that there are two different equlibrium points).

(b) By our establishment at the beginning of the proof and by Lemma 21 (b) and (c), equation (51) is globally $p$-periodic with $p \geq 3$ only if either (57) and (58) are satisfied, or

$$
\frac{a_{2}-b_{2} c_{i}}{b_{0}+\left(b_{1}+b_{2}\right) c_{i}}=-1, \quad i=1,2,
$$

and there is an integers $1 \leq k_{1}, k_{2}<\frac{p}{2}$ such that

$$
\frac{a_{1}-b_{1} c_{1}}{b_{0}+\left(b_{1}+b_{2}\right) c_{1}}=\cos \left(\frac{2 \pi}{p} k_{i}\right), \quad i=1,2 .
$$

By (a), we can study just the second condition. (59) gives that

$$
a_{2}=-b_{0}-b_{1} c_{i}, \quad i=1,2,
$$

and therefore $b_{1}=0$ comes from $c_{1} \neq c_{2}$. This means that $a_{2}=-b_{0} . b_{2} \neq 0$ is also satisfied (otherwise $\left|b_{1}\right|+\left|b_{2}\right| \neq 0$ ). We have thus proved (54).

To take into account (54) we have

$$
\begin{aligned}
& c_{1}=\frac{-\left(2 b_{0}-a_{1}\right)+\sqrt{\left(2 b_{0}-a_{1}\right)^{2}+4 a_{0} b_{2}}}{2 b_{2}} \\
& c_{2}=\frac{-\left(2 b_{0}-a_{1}\right)-\sqrt{\left(2 b_{0}-a_{1}\right)^{2}+4 a_{0} b_{2}}}{2 b_{2}},
\end{aligned}
$$

or conversely. Since there exist two real equilibrium points

$$
\left(2 b_{0}-a_{1}\right)^{2}+4 a_{0} b_{2}>0,
$$


which is the first part of (55). The second part of (55) is valid, by

$$
b_{0}+b_{2} c_{i} \neq 0, \quad i=1,2 .
$$

The middle condition in (52) holds, since $b_{2} \neq 0$. The first condition in (52) is also satisfied: if $a_{1} \neq 0$, then

$$
\operatorname{det}\left(\begin{array}{cc}
a_{1} & -b_{0} \\
0 & b_{2}
\end{array}\right) \neq 0
$$

while if $a_{1}=0$, then

$$
\operatorname{det}\left(\begin{array}{cc}
a_{0} & -b_{0} \\
b_{0} & b_{2}
\end{array}\right)=a_{0} b_{2}+b_{0}^{2}>0
$$

because of (61).

Finally, (56) comes from (60) and

$$
\frac{a_{1}}{b_{0}+b_{2} c_{1}} \neq \frac{a_{1}}{b_{0}+b_{2} c_{2}} .
$$

The proof is now complete.

We immediately have from (56) that if equation (51) is globally $p$-periodic with $p \geq 3$, then $p \geq 5$. In the next result the case $p=5$ will be investigated by the help of Theorem 5 .

Theorem 38 If equation (51) has the form

$$
x(n)=\frac{\frac{a_{1}^{2}-b_{0}^{2}+b_{0} a_{1}}{b_{2}}+a_{1} x(n-1)-b_{0} x(n-2)}{b_{0}+b_{2} x(n-2)}, \quad n \geq 0,
$$

where $a_{1} \neq 0$ and $b_{2} \neq 0$, then it is globally 5-periodic. Furthermore, (62) has two equilibrium points.

Proof. By applying Theorem 37 (b), we get equation (62), since

$$
\cos \left(\frac{2 \pi}{5}\right)=\frac{1}{4} \sqrt{5}-\frac{1}{4}, \quad \cos \left(\frac{4 \pi}{5}\right)=-\frac{1}{4} \sqrt{5}-\frac{1}{4}
$$

$a_{1} \neq 0$ and $b_{2} \neq 0$ imply that (52) is satisfied.

By using the recursion defined in (7) and (8), we have for all $(s, t) \in D_{a d}$ that

$$
\begin{gathered}
f_{0}(t, s)=\frac{1}{b_{2}\left(b_{0}+s b_{2}\right)}\left(a_{1}^{2}+a_{1} b_{0}+t b_{2} a_{1}-b_{0}^{2}-s b_{2} b_{0}\right), \\
f_{1}(t, s)=\frac{2 a_{1}^{2} b_{0}+a_{1}^{3}-b_{0}^{3}+s a_{1}^{2} b_{2}-s b_{0}^{2} b_{2}+t a_{1}^{2} b_{2}-t b_{0}^{2} b_{2}-s t b_{0} b_{2}^{2}}{b_{2}\left(b_{0}+s b_{2}\right)\left(b_{0}+t b_{2}\right)}, \\
f_{2}(t, s)=\frac{1}{b_{2}\left(b_{0}+t b_{2}\right)}\left(a_{1}^{2}+a_{1} b_{0}+s b_{2} a_{1}-b_{0}^{2}-t b_{2} b_{0}\right), \\
f_{3}(t, s)=s,
\end{gathered}
$$

which give the first part of the result by Theorem 5 .

It is easy to check that there are two equilibrium points.

The proof is complete. 


\subsection{Existence of periodic solutions}

Consider the equation

$$
x(n)=\frac{\beta x(n-1)+x(n-2)}{x(n-2)+D x(n-3)}, \quad n \geq 0,
$$

where $\beta, D \in \mathbb{R}$. The study of the existence of a periodic solution of (63) with prime period 2 is motivated by the Conjecture 3 in [8]. The next result gives a necessary and sufficient condition for this problem.

Theorem 39 Equation (63) has a periodic solution with prime period 2 if and only if either

$$
\beta=0, \quad D=1
$$

or

$$
\left.\begin{array}{c}
(D-1)(1-\beta)((D-1)(1-\beta)-4 \beta)>0 \\
(D+1)^{2} \neq(D-1)(1-\beta)((D-1)(1-\beta)-4 \beta)
\end{array}\right\} .
$$

Proof. By Theorem 28, we have to investigate the solvability of the system of equations

$$
y_{2}=\frac{\beta y_{1}+y_{2}}{y_{2}+D y_{1}}, \quad y_{1}=\frac{\beta y_{2}+y_{1}}{y_{1}+D y_{2}}, \quad y_{1}, y_{2} \in \mathbb{R} .
$$

If $\beta=1$, then it is easy to check that (65) has a solution if and only if $D \neq-1$, but in these cases

$$
y_{1}=y_{2}=\frac{2}{1+D}
$$

and therefore there are no solutions of (63) with prime period 2 .

Suppose $\beta \neq 1$. By applying a simple calculation we get from (65) that

$$
y_{2}+y_{1}=1-\beta
$$

and hence

$$
(1-D) y_{1}^{2}+(D-1)(1-\beta) y_{1}-\beta+\beta^{2}=0 .
$$

Suppose $D=1$. Then (67) has a solution, bearing in mind that $\beta \neq 1$, if and only if $\beta=0$. In this case (67) holds for every $y_{1} \in \mathbb{R}$, and therefore (66) implies that the vectors

$$
(a, 1-a, a) \in \mathbb{R}^{3}, \quad a \neq \frac{1}{2}
$$

are 2-periodic initial vectors (the prime period is 2) of the equation

$$
x(n)=\frac{x(n-2)}{x(n-2)+x(n-3)}, \quad n \geq 0 .
$$

Suppose $D \neq 1$. Then (67) has a solutions if and only if

$$
(D-1)(1-\beta)((D-1)(1-\beta)-4 \beta) \geq 0 .
$$


Since we are interested in 2-periodic solutions with prime period $2, t_{1} \neq t_{2}$, and hence

$$
(D-1)(1-\beta)((D-1)(1-\beta)-4 \beta)>0,
$$

which is the first condition in (64). The second condition comes from (65) because of

$$
y_{2}+D y_{1} \neq 0, \quad y_{1}+D y_{2} \neq 0 .
$$

It follows that under the conditions (64) the vectors

$$
\left(y_{1}, y_{2}, y_{1}\right) \in \mathbb{R}^{3}
$$

are 2-periodic initial vectors (the prime period is 2 ) of equation (63), where $y_{1}$ and $y_{2}$ are solutions of (67).

The proof is complete.

Remark 40 (a) For example, if $\beta=D=2$, then the first condition in (64) is satisfied, but the second one is not.

(b) By using Theorem 26 and Theorem 27, it can be seen that there exist real numbers $\beta$ and $D$ such that the corresponding equations of the form (63) have a periodic solution with either prime period 3 or with prime period 4 .

$(0,2,-2) \in \mathbb{R}^{3}$ is a 3 -periodic initial vector of the equation

$$
x(n)=\frac{x(n-1)+x(n-2)}{x(n-2)+\frac{1}{2} x(n-3)}, \quad n \geq 0 .
$$

It is easy to check that if $a$ is the unique real root of the equation $(a \approx 1,5437)$

$$
a^{5}-2 a^{2}-4=0,
$$

then $\left(-1,0,-\frac{a^{2}}{2}, a\right) \in \mathbb{R}^{4}$ is a 4-periodic initial vector of the equation

$$
x(n)=\frac{a x(n-1)+x(n-2)}{x(n-2)+\frac{a^{2}}{2} x(n-3)}, \quad n \geq 0 .
$$

Next, motivated by the paper [28], we examine the difference equation

$$
x(n)=1+\frac{x(n-k)}{x(n-m)}, \quad n \geq 0
$$

where $1 \leq k<m$ are integers. It can be verified easily that equation (68) has a constant solution $x(n)=2(n \geq 0)$, and there are no 2-periodic solutions with prime period 2. Now we study the existence of 3-periodic solutions with prime period 3.

Theorem 41 Equation (68) has a 3-periodic solutions with prime period 3 if and only if $3 \nmid k, 3 \nmid m$ and $3 \mid k+m$. 
Proof. If $m=2$, then Theorem 26 can be applied, otherwise Theorem 28 can be used. We obtain that there are 4 possibilities for the systems of equations (36) or (37):

If $3 \mid k+m$, then

$$
\left.\begin{array}{l}
b c=b+a \\
b a=a+c \\
a c=c+b
\end{array}\right\}
$$

and in the other cases

$$
\left.\left.\left.\begin{array}{l}
a=2 \\
b=2 \\
c=2
\end{array}\right\}, \quad \begin{array}{l}
a c=a+c \\
b c=c+b \\
a b=b+a
\end{array}\right\}, \begin{array}{l}
c^{2}=a+c \\
b^{2}=c+b \\
c^{2}=b+a
\end{array}\right\},
$$

where $a, b, c \in \mathbb{R} \backslash\{0\}$.

(69) leads to the equation

$$
r(b):=b^{3}+b^{2}-2 b-1=0,
$$

which has 3 real roots, since $r(-2)<0, r(-1)>0, r(0)<0$ and $r(2)>0$. Numerically, the roots

$$
b_{1} \approx-1,8019, \quad b_{2} \approx-0,44504, \quad b_{3} \approx 1,247 .
$$

We can see that if $3 \mid k+m$, then equation (68) has a 3-periodic solutions with prime period 3 .

Now we consider the second equation in (70). Some elementary calculations show that the only solutions $a=b=c=2$, and thus there are no 3-periodic solutions of equation (68) with prime period 3 .

Finally, from the third equation in (70) we have

$$
r(b):=b^{6}-2 b^{5}+2 b^{3}-b^{2}+1=0 .
$$

In this case

$$
r^{\prime}(b)=b(b-1)^{2}\left(6 b^{2}+2 b-2\right), \quad b \in \mathbb{R},
$$

and this insures that $r(b)>0(b \in \mathbb{R})$, and thus there are no 3 -periodic solutions of equation (68) with prime period 3.

The proof is complete.

Remark 42 In [28] the author gives a subclass of equation (68) which have periodic solutions, but these equations are different from the equations in the previous theorem.

Finally, we examine the difference equation

$$
x(n)=\frac{\beta x(n-1)}{1+x(n-2)}, \quad n \geq 0,
$$

where $\beta$ is a real number different from 0 . It is easy to check that the constant or 1-periodic solutions of equation (71) are $x(n)=0$ and $x(n)=\beta-1(n \geq-2)$. 
Theorem 43 (a) Equation (71) has a 2-periodic solution with prime period 2 if and only if $-1<\beta<\frac{1}{3}$.

(b) For every $\beta \in \mathbb{R} \backslash\{0\}$ equation (71) has a 3 -periodic solution with prime period 3 .

Proof. (a) By Theorem 26, $\left(y_{2}, y_{1}\right) \in \mathbb{R}^{2}$ is a 2-periodic initial vector of equation (71) if and only if

$$
y_{2}=\frac{\beta y_{1}}{1+y_{2}}, \quad y_{1}=\frac{\beta y_{2}}{1+y_{1}}
$$

are satisfied. We can deduce from this that either $y_{1}=y_{2}$ or $y_{2}=-1-\beta-y_{1}$. Since we are looking for 2-periodic solutions with prime period 2, the following second order equation is obtained

$$
y_{1}^{2}+(1+\beta) y_{1}+\beta+\beta^{2}=0 .
$$

By analyzing (72), we have the result.

(b) Theorem 27 can be applied: $\left(y_{2}, y_{1}\right) \in \mathbb{R}^{2}$ is a 3 -periodic initial vector of equation (71) if and only if

$$
y_{3}=\frac{\beta y_{1}}{1+y_{2}}, \quad y_{2}=\frac{\beta y_{3}}{1+y_{1}}, \quad y_{1}=\frac{\beta y_{2}}{1+y_{3}}
$$

are satisfied. The first equation implies

$$
y_{1}=\frac{1}{\beta} y_{3}\left(1+y_{2}\right)
$$

which gives that

$$
\left.\begin{array}{c}
y_{3}=\frac{-\beta y_{2}}{y_{2}^{2}+y_{2}-\beta^{2}} \\
y_{3}+y_{3} y_{2}+y_{3}^{2}+y_{3}^{2} y_{2}=\beta^{2} y_{2}
\end{array}\right\}
$$

Therefore, some easy calculations show that

$$
\beta y_{2}^{3}+\left(\beta^{2}+\beta+1\right) y_{2}^{2}+\left(1-\beta^{3}\right) y_{2}-\beta^{2}\left(\beta^{2}+\beta+1\right)=0 .
$$

Since (73) is a third order equation, it has a real root $\alpha$. Moreover, $\alpha \neq 0$, $\alpha \neq \beta-1$, and $\alpha^{2}+\alpha-\beta^{2} \neq 0$, and hence

$$
\left(\alpha, \frac{-\alpha(1+\alpha)}{\alpha^{2}+\alpha-\beta^{2}}\right)
$$

is a nonconstant 3-periodic initial vector of equation (71).

The proof is complete.

Remark 44 To examine the p-periodic $(p>3)$ solutions of equation (71), Theorem 27 shows that we have to study the solvability of the following system of polynomial equations in p-variables

$$
\left.\begin{array}{c}
y_{p}\left(1+y_{2}\right)-\beta y_{1}=0 \\
y_{p-1}\left(1+y_{1}\right)-\beta y_{p}=0 \\
\vdots \\
y_{1}\left(1+y_{3}\right)-\beta y_{2}=0
\end{array}\right\} \text {. }
$$


There are a lot of different methods to study (7/4). We mention just two: algorithms for Gröbner bases in computer algebra (see [32]); homotopy and degree theory (see [21]).

\section{Preliminary results and the proof of the main results}

Proof of Theorem 26. (a) Suppose $\varphi=(\varphi(-s), \ldots, \varphi(-1)) \in D_{a d}$ is an $s$-periodic initial vector of (1), and let $k \geq 1$ be an integer. Then

$$
\begin{gathered}
\varphi(-s)=x(\varphi)(k s-s) \\
=h_{k s-s}(x(\varphi)(k s-s-1), \ldots, x(\varphi)(k s-s-s)) \\
=h_{k s-s}(\varphi(-1), \ldots, \varphi(-s))
\end{gathered}
$$

and for $1 \leq i \leq s-1$

$$
\begin{gathered}
\varphi(-i)=x(\varphi)(k s-i)=h_{k s-i}(x(\varphi)(k s-i-1), \ldots, x(\varphi)(k s-i-s)) \\
=h_{k s-i}(x(\varphi)(k s-(i+1)), \ldots, x(\varphi)(k s-s), x(\varphi)((k-1) s-1)), \\
x(\varphi)((k-1) s-2), \ldots, x(\varphi)((k-1) s-i))) \\
=h_{k s-i}(\varphi(-i-1), \ldots, \varphi(-s), \varphi(-1), \varphi(-2), \ldots, \varphi(-i)),
\end{gathered}
$$

and therefore $\varphi$ satisfies (36).

(b) Conversely, suppose $\varphi:=\left(y_{1}, \ldots, y_{s}\right) \in X^{s}$ is a solution of (36) for all $k \geq 1$. If $n \geq-s$ is an integer, then $n$ has the unique form

$$
n=k s-i \text { with } k \geq 0 \text { and } 1 \leq i \leq s .
$$

Define

$$
x(n):=y_{i} .
$$

It is enough to prove that $(x(n))_{n \geq-s}$ is a solution of (1). If $n=k s-s$ with $k \geq 1$, then by (36),

$$
\begin{gathered}
x(n)=y_{s}=h_{k s-s}\left(y_{1}, y_{2}, y_{3}, \ldots, y_{s-1}, y_{s}\right) \\
=h_{n}(x(n-1), x(n-2), x(n-3), \ldots, x(n-s)),
\end{gathered}
$$

while if $n=k s-i$ with $k \geq 1$ and $1 \leq i \leq s-1$, then (36) shows that

$$
\begin{gathered}
x(n)=y_{i}=h_{k s-i}\left(y_{i+1}, y_{i+2}, \ldots, y_{s}, y_{1}, \ldots, y_{i}\right) \\
=h_{n}(x(n-1), x(n-2), \ldots, x(n-(s-i)), x(n-(s-i+1), \ldots, x(n-s)),
\end{gathered}
$$

and this completes the proof. 
Before turning to the proof of our results in the case $p \neq s$, we show that the problem is facilitated by the introduction of a new equation

$$
z(n)=g_{n}(z(n-1), \ldots, z(n-p)), \quad n \geq 0
$$

corresponding to (1), where $g_{n}$ is a function from a subset of $X^{p}$ into $X$ for every $n \geq 0$. We shall see that it is enough to study the existence of $p$-periodic solutions of equation (75) which can be handled by Theorem 26. $D_{a d}^{g}$ denotes the set of all admissible initial vectors $\psi=(\psi(-p), \ldots, \psi(-1)) \in X^{p}$ for equation (75).

Theorem 45 Consider equation (1). Suppose $1 \leq p<s$ is an integer, and let $u:=\left[\frac{s}{p}\right]$ and $v:=s-u p(0 \leq v \leq p-1)$. Let

$$
\begin{gathered}
g_{n}\left(t_{1}, \ldots, t_{p}\right) \\
:=\left\{\begin{array}{c}
h_{n}\left(t_{1}, \ldots, t_{p}, t_{1}, \ldots, t_{p}, \ldots, t_{1}, \ldots, t_{p}\right), \quad v=0 \\
h_{n}\left(t_{1}, \ldots, t_{p}, t_{1}, \ldots, t_{p}, \ldots, t_{1}, \ldots, t_{p}, t_{1}, \ldots, t_{v}\right), \quad v \neq 0
\end{array}\right.
\end{gathered}
$$

for all $n \geq 0$, and consider equation (75) with functions defined in (76).

(a) If $\varphi=(\varphi(-s), \ldots, \varphi(-1)) \in D_{a d}$ is a p-periodic initial vector of (1), then $\psi:=(\varphi(-p), \ldots, \varphi(-1)) \in D_{a d}^{g}$ and $\psi$ is a $p$-periodic initial vector of the equation (75).

(b) If $\psi=(\psi(-p), \ldots, \psi(-1)) \in D_{a d}^{g}$ is a p-periodic initial vector of the equation (75), then $\varphi=(\varphi(-s), \ldots, \varphi(-1))$ defined by $\varphi(i):=\psi(j)$ if $i \equiv j$ $(\bmod p)(-s \leq i \leq-1,-p \leq j \leq-1)$ belongs to $D_{a d}$ and $\varphi$ is a p-periodic initial vector of (1).

Proof. (a) It is enough to check that

$$
z(n):=x(\varphi)(n), \quad n \geq-p
$$

is the solution of (75) satisfying the initial condition $\psi$. Obviously,

$$
z(i)=\varphi(i), \quad-p \leq i \leq-1 .
$$

Since $\varphi$ is a $p$-periodic initial vector of $(1)$,

$$
\begin{gathered}
z(n)=x(\varphi)(n)=h_{n}(x(\varphi)(n-1), \ldots, x(\varphi)(n-s)) \\
=h_{n}(x(\varphi)(n-1), \ldots, x(\varphi)(n-p), x(\varphi)(n-1), \ldots, x(\varphi)(n-p), \\
\ldots, x(\varphi)(n-1), \ldots, x(\varphi)(n-p), x(\varphi)(n-1), \ldots, x(\varphi)(n-v)) \\
=g_{n}(x(\varphi)(n-1), \ldots, x(\varphi)(n-p))=g_{n}(z(n-1), \ldots, z(n-p)), \quad n \geq 0 .
\end{gathered}
$$

(b) Now suppose $\psi=(\psi(-p), \ldots, \psi(-1)) \in D_{a d}^{g}$ is a $p$-periodic initial vector of the equation (75), and let $\varphi=(\varphi(-s), \ldots, \varphi(-1)) \in X^{s}$, where $\varphi(i):=\psi(j)$ if $i \equiv j(\bmod p)(i=-s, \ldots,-1)$. Set

$$
z(\psi)(i):=\psi(j) \text { if } i \equiv j(\bmod p), \quad i=-s, \ldots,-1 .
$$


By the definition of $\varphi$, it is enough to verify that

$$
x(n):=z(\psi)(n), \quad n \geq-s
$$

is the solution of (1) satisfying the initial condition $\varphi$. Since $\psi$ is a $p$-periodic initial vector of $(75)$,

$$
\begin{gathered}
x(n)=z(\psi)(n)=g_{n}(z(\psi)(n-1), \ldots, z(\psi)(n-p)) \\
h_{n}(z(\psi)(n-1), \ldots, z(\psi)(n-p), z(\psi)(n-1), \ldots, z(\psi)(n-p), \\
\ldots, z(\psi)(n-1), \ldots, z(\psi)(n-p), z(\psi)(n-1), \ldots, z(\psi)(n-v)) \\
=h_{n}(z(\psi)(n-1), \ldots, z(\psi)(n-p), z(\psi)(n-p-1), \ldots, z(\psi)(n-2 p), \\
\ldots, z(\psi)(n-(u-1) p-1), \ldots, z(\psi)(n-u p),) \\
z(\psi)(n-u p-1), \ldots, z(\psi)(n-u p-v)) \\
=h_{n}(x(n-1), \ldots, x(n-s)), \quad n \geq 0 .
\end{gathered}
$$

Theorem 46 Consider equation (1). Suppose $p>s$ is an integer. Let

$$
g_{n}\left(t_{1}, \ldots, t_{s}, \ldots, t_{p}\right):=h_{n}\left(t_{1}, \ldots, t_{s}\right)
$$

for all $n \geq 0$, and consider the equation (75) with functions defined in (77). then

(a) If $\varphi=(\varphi(-s), \ldots, \varphi(-1)) \in D_{a d}$ is a p-periodic initial vector of (1),

$$
\psi:=(x(\varphi)(0), \ldots, x(\varphi)(p-s-1), \varphi(-s), \ldots, \varphi(-1)) \in D_{a d}^{g},
$$

and $\psi$ is a p-periodic initial vector of the equation (75).

(b) If $\psi:=(\psi(-p), \ldots, \psi(-1)) \in D_{a d}^{g}$ is a $p$-periodic initial vector of the equation (75), then $\varphi=(\psi(-s), \ldots, \psi(-1)) \in D_{a d}$ is a p-periodic initial vector of (1).

Proof. This follows by an argument similar to that for the previous theorem, and we omit the details.

Now we are able to prove Theorem 28 and Theorem 27 .

Proofs of Theorem 28 and Theorem 27. Theorem 28 (a) follows from Theorem 45 (a) and Theorem 26 (a).

Theorem 28 (b) follows from Theorem 45 (b) and Theorem 26 (b).

Theorem 27 (a) comes from Theorem 46 (a) and Theorem 26 (a).

Theorem 27 (b) comes from Theorem 46 (b) and Theorem 26 (b).

Proof of Theorem 5. By Theorem 26 and Theorem 27, equation (6) is globally $p$-periodic on $A$ if and only if

$$
f_{i}\left(y_{1}, \ldots, y_{s}\right)=y_{p-i}, \quad p-s \leq i \leq p-1
$$

for all $\left(y_{s}, \ldots, y_{1}\right) \in A$.

(a) Choose $i=p-s$ in (78). 
(b) Only the task of confirming the following assertion remains: If $A$ is invariant and

$$
f_{p-s}\left(y_{1}, \ldots, y_{s}\right)=y_{s}
$$

for all $\left(y_{s}, \ldots, y_{1}\right) \in A$, then (78) holds.

We get from (8) with $k=p-s$ that

$$
f_{i}\left(y_{1}, \ldots, y_{s}\right)=f_{p-s}\left(f_{i-(p-s)-1}\left(y_{1}, \ldots, y_{s}\right), \ldots, f_{i-(p-s)-s}\left(y_{1}, \ldots, y_{s}\right)\right)
$$

for all $i \geq p-s$. Since $A$ is invariant, $\left(y_{s}, \ldots, y_{1}\right) \in A$ implies

$$
\left(f_{i-(p-s)-s}\left(y_{1}, \ldots, y_{s}\right), \ldots, f_{i-(p-s)-1}\left(y_{1}, \ldots, y_{s}\right)\right) \in A
$$

for every $p-s \leq i \leq p-1$, and therefore (80) and (79) yield

$$
f_{i}\left(y_{1}, \ldots, y_{s}\right)=f_{i-(p-s)-s}\left(y_{1}, \ldots, y_{s}\right), \quad p-s \leq i \leq p-1 .
$$

Finally, (7) gives

$$
f_{i-(p-s)-s}\left(y_{1}, \ldots, y_{s}\right)=y_{p-i}, \quad p-s \leq i \leq p-1 .
$$

The proof is complete.

Proof of Theorem 8. It is an easy consequence of Theorem 28.

The following results are needed to prove Theorem 12 .

Lemma 47 Consider equation (40). Let $p \geq s$ be a positive integer. Suppose $\varphi=\left(a_{s}, \ldots, a_{1}\right)$ is an interior point of $D_{a d}$, and let $U \subset D_{a d}$ be a neigbourhood of $\varphi$. Define the set

$$
A_{U}:=\left\{\left(f_{i-s}\left(y_{1}, \ldots, y_{s}\right), \ldots, f_{i-1}\left(y_{1}, \ldots, y_{s}\right)\right) \mid\left(y_{s}, \ldots, y_{1}\right) \in U, \quad i \geq 0\right\} .
$$

(a) $A_{U}$ is an invariant subset of $D_{a d}$.

The following statements are equivalent:

(b) Equation (14) is globally p-periodic on $U$.

(c) Equation (14) is globally p-periodic on $A_{U}$.

Proof. (a) It is obvious.

(c) $\Longrightarrow(\mathrm{b})$ It comes from $U \subset A_{U}$.

(b) $\Longrightarrow(\mathrm{c})$ Equation (14) is Volterra type.

Lemma 48 Let $X$ be a real normed space, and let $p \geq s$ be a positive integer. We consider the higher order linear difference equation

$$
x(n)=a+\sum_{i=1}^{s} A_{i}(x(n-i)-a), \quad n \geq 0,
$$

where $s \geq 1$ is a given integer, $a \in X$, and $A_{i}$ is a continuous linear map from $X$ into itself for every $1 \leq i \leq s$. If there is a neigbourhood $U$ of $\varphi_{e}=(a, \ldots, a) \in$ $X^{s}$ such that equation (81) is globally p-periodic on $U$, then equation (81) is globally p-periodic. 
Proof. First, $a=0$ is assumed, that is we study the homogeneous linear equation

$$
y(n)=\sum_{i=1}^{s} A_{i} y(n-i), \quad n \geq 0
$$

associated with the equation $(81)$. Let $\varphi=\left(y_{s}, \ldots, y_{1}\right) \in X^{s}$. Then there is a vector $\psi=\left(z_{s}, \ldots, z_{1}\right) \in U$ and a real number $c$ such that $\varphi=c \psi$. Since $\psi$ is a $p$-periodic initial vector and equation (82) is homogeneous

$$
y(\varphi)(n+p)=c y(\psi)(n+p)=c y(\psi)(n)=y(\varphi)(n), \quad n \geq 0 .
$$

Since $x\left(\varphi_{e}\right)=(a)_{n>-s}$ is a (p-periodic) solution of equation (81), we have for each $\varphi=\left(y_{s}, \ldots, y_{1}\right) \in X^{s}$ that

$$
x(\varphi)=x\left(\varphi_{e}\right)+y\left(\varphi-\varphi_{e}\right),
$$

where $y\left(\varphi-\varphi_{e}\right)$ is the solution of equation (82) determined by the initial value $\varphi-\varphi_{e}$. This shows that every solution of equation (82) determined by an initial value from $U_{a}:=U-a$ is $p$-periodic. Since $U_{a}$ is a neigbourhood of $(0, \ldots, 0) \in X^{s}$, the result follows from the first part of the proof and (83).

Lemma 49 Consider equation (40), let $a \in X$ be an equilibrium point of equation (14), and let $h$ be differentiable at $\varphi_{e}=(a, \ldots, a) \in X^{s}$. Suppose $\varphi_{e}$ is an interior point of $D_{a d}$, and let $U \subset D_{a d}$ be a neighbourhood of $\varphi_{e}$. Consider the recursive sequence $\left(f_{i}\right)_{i \geq-s}$ defined by (7) and (8). Denote $\left(g_{i}\right)_{i \geq-s}$ the analogue recursive sequence coming from the linearized equation of (14) around a.

(a) $f_{i}$ is differentiable at $\varphi_{e}$ and $f_{i}\left(\varphi_{e}\right)=$ a for every $i \geq-s$.

(b) $g_{i}$ is the first degree Taylor polynomial of $f_{i}$ around $\varphi_{e}$ for every $i \geq-s$.

Proof. (a) $f_{i}(-s \leq i \leq-1)$ is trivially differentiable at $\varphi_{e}$. Now the result follows from (8), the differentiability of $h$ at $\varphi_{e}$, and the chain rule by an easy induction argument.

(b) We prove this by induction on $i$. It is obvious that $g_{i}$ is the first degree Taylor polynomial of $f_{i}$ around $\varphi_{e}$ for every $-s \leq i \leq-1$. Suppose then that $i$ is a nonnegative integer such that the result holds for $i-s, \ldots, i-1$. The form of the linearized equation (15), (8), the induction hypothesis and the second part of (a) imply that

$$
\begin{gathered}
g_{i}\left(y_{1}, \ldots, y_{s}\right)=a+\sum_{j=1}^{s} d_{j} h\left(\varphi_{e}\right)\left(g_{i-j}\left(y_{1}, \ldots, y_{s}\right)-a\right) \\
=a+\sum_{j=1}^{s} d_{j} h\left(\varphi_{e}\right)\left(\sum_{k=1}^{s} d_{k} f_{i-j}\left(\varphi_{e}\right)\left(y_{k}-a\right)\right) \\
=a+\sum_{k=1}^{s}\left(\sum_{j=1}^{s} d_{j} h\left(\varphi_{e}\right) \circ d_{k} f_{i-j}\left(\varphi_{e}\right)\right)\left(y_{k}-a\right), \quad\left(y_{1}, \ldots, y_{s}\right) \in X^{s} .
\end{gathered}
$$


It remains to prove that

$$
d_{k} f_{i}\left(\varphi_{e}\right)=\sum_{j=1}^{s} d_{j} h\left(\varphi_{e}\right) \circ d_{k} f_{i-j}\left(\varphi_{e}\right), \quad 1 \leq k \leq s,
$$

but this comes from the first part of (a) and the chain rule.

Now we are able to prove Theorem 12.

Proof of Theorem 12. By Remark 13, it is enough to prove that equation (15) is globally $p$-periodic.

We shall use the sequences $\left(f_{i}\right)_{i \geq-s}$ and $\left(g_{i}\right)_{i \geq-s}$ introduced in Lemma 49.

By the equivalence of Lemma 47 (b) and (c), we can suppose that every solution of equation (14) determined by an initial value from the set

$$
A_{U}:=\left\{\left(f_{i-s}\left(y_{1}, \ldots, y_{s}\right), \ldots, f_{i-1}\left(y_{1}, \ldots, y_{s}\right)\right) \mid\left(y_{s}, \ldots, y_{1}\right) \in U, \quad i \geq 0\right\} .
$$

is $p$-periodic. We know from Lemma 47 (a) that $A_{U}$ is an invariant subset of $D_{a d}$, and therefore the necessary part of Theorem 5 can be applied:

$$
f_{p-s}\left(y_{1}, y_{2}, \ldots, y_{s}\right)=y_{s}
$$

for all $\left(y_{s}, y_{s-1}, \ldots, y_{1}\right) \in A_{U}$.

From this and from Lemma 49 (b) we get

$$
g_{p-s}\left(y_{1}, y_{2}, \ldots, y_{s}\right)=y_{s}
$$

for all $\left(y_{s}, y_{s-1}, \ldots, y_{1}\right) \in A_{U}$. Now the sufficient part of Theorem 5 can be applied to the linearized equation (15): equation (15) is globally $p$-periodic on $A_{U}$. Consequently, the result follows from Lemma 48.

The proof is complete.

Proof of Theorem 14. (a) It is easy to see that equation (17) has not got an equilibrium point if and only if

$$
\sum_{i=1}^{s} A_{i}=1 \quad \text { and } \quad b \neq 0 .
$$

Therefore we should prove the next statement: if the conditions in (84) are satisfied, then there exists an unbounded solution of equation (17). Assume $s=1$. Then $x(n)=(n+2) b(n \geq-1)$ is an unbounded solution of equation

$$
x(n)=x(n-1)+b, \quad n \geq 0 .
$$

We complete the proof by induction on $s$. Suppose $s \geq 2$ is an integer such that every equation

$$
x(n)=\sum_{i=1}^{s-1} A_{i} x(n-i)+b, \quad n \geq 0
$$


satisfying the stated hypotheses

$$
\sum_{i=1}^{s-1} A_{i}=1 \quad \text { and } \quad b \neq 0
$$

has an unbounded solution, and consider equation

$$
x(n)=\sum_{i=1}^{s} A_{i} x(n-i)+b, \quad n \geq 0
$$

with (84).

It is not hard to check that if

$$
c:=\sum_{i=1}^{s} i A_{i} \neq 0,
$$

then $x(n)=n \frac{b}{c}(n \geq-s)$ is an unbounded solution of equation (85).

Suppose $c=0$. By introducing

$$
y(n):=x(n)-x(n-1), \quad n \geq-s+1,
$$

we have

$$
\begin{aligned}
x(n) & =-\sum_{i=1}^{s} A_{i}\left(-x(n)+\sum_{j=0}^{i-1}(x(n-j)-x(n-j-1))\right)+b \\
& =x(n) \sum_{i=1}^{s} A_{i}-\sum_{j=0}^{s-1}\left(\sum_{i=j+1}^{s} A_{i}\right) y(n-j)+b, \quad n \geq 0 .
\end{aligned}
$$

It follows from this, by using the first condition in (84), that

$$
y(n)=-\sum_{j=1}^{s-1}\left(\sum_{i=j+1}^{s} A_{i}\right) y(n-j)+b, \quad n \geq 0 .
$$

Since $c=0$

$$
\begin{gathered}
-\sum_{j=1}^{s-1}\left(\sum_{i=j+1}^{s} A_{i}\right)=-\sum_{j=0}^{s-1}\left(\sum_{i=j+1}^{s} A_{i}\right)+1 \\
=-\sum_{i=1}^{s}\left(A_{i} \sum_{j=0}^{i-1} 1\right)+1=-c+1=1,
\end{gathered}
$$

and therefore the induction hypothesis shows that equation (87) has an unbounded solution. Hence, by (86), equation (85) also has an unbounded solution. 
(b) It follows from (a), by Remark 13 (a) and (b).

The proof is complete.

Proof of Lemma 21. The structure of the fundamental solutions of equation (33) (see [12]) shows that the global periodicity of (33) implies that the characteristic equation of (33)

$$
\lambda^{2}-A_{1} \lambda-A_{2}=0
$$

has two different characteristic roots $\lambda_{1}$ and $\lambda_{2}$ with absolute value 1 . it follows that either

$$
\lambda_{1}=1 \quad \text { and } \quad \lambda_{2}=-1
$$

or

$$
\lambda_{1}, \lambda_{2} \in \mathbb{C}, \quad \lambda_{1}=\overline{\lambda_{2}} \quad \text { and } \quad\left|\lambda_{1}\right|=1 .
$$

In the first case

$$
A_{1}=0 \quad \text { and } \quad A_{2}=1 \text {, }
$$

while in the second case

$$
\left|A_{1}\right|<2 \text { and } A_{2}=-1 .
$$

If (88) holds, then obviously, equation (33) is globally 2-periodic. Conversely, it is easy to check that if equation (33) is globally 2-periodic, then (88) holds.

Suppose (89) is satisfied. If equation (33) is globally $p$-periodic with an integer $p \geq 2$, then there exists a $p$-periodic solution, and therefore an immediate consequence of Theorem 6 and 7 in [20] that $p \geq 3$ and

$$
A_{1}=2 \cos \left(\frac{2 \pi}{p} k\right), \quad 1 \leq k<\frac{p}{2} \quad \text { is an integer. }
$$

It now remained to prove that if $A_{2}=-1$ and (90) holds, then (33) is globally $p$-periodic. It also follows from Theorem 7 in [20] that in this case

$$
\left(\cos \left(\frac{4 \pi}{p} k\right), \cos \left(\frac{2 \pi}{p} k\right)\right)
$$

and

$$
\left(-\sin \left(\frac{4 \pi}{p} k\right),-\sin \left(\frac{2 \pi}{p} k\right)\right)
$$

are $p$-periodic initial vectors of (33). Since these vectors are independent, the result follows.

The proof is complete.

Proof of Theorem 3. By Theorem 5, equation (4) is globally $(s+1)$-periodic if and only if

$$
f\left(f\left(y_{1}\right) \ldots f\left(y_{s}\right)\right) f\left(y_{1}\right) \ldots f\left(y_{s-1}\right)=y_{s}, \quad\left(y_{s}, y_{s-1}, \ldots, y_{1}\right) \in G^{s} .
$$

Choose $y_{1}=\ldots=y_{s-1}=e$ in (91):

$$
f\left(c f\left(y_{s}\right)\right)=y_{s} c^{-1}, \quad y_{s} \in G,
$$


where $c:=f(e)^{s-1}$, and therefore $f$ is a surjection.

Since $f$ is surjective, there exist $y_{1}, \ldots, y_{s-1} \in G$ such that

$$
f\left(y_{1}\right) \ldots f\left(y_{s-1}\right)=e,
$$

and then because of (91)

$$
f\left(f\left(y_{s}\right)\right)=y_{s}, \quad y_{s} \in G,
$$

which implies that $f$ is invertible and $f^{-1}=f$.

With the help of the surjectivity of $f$ again, for every $x \in G$ there exist $y_{1}, \ldots, y_{s-1} \in G$ such that

$$
f\left(y_{1}\right) \ldots f\left(y_{s-1}\right)=x
$$

and therefore (91) and $f^{-1}=f$ yield

$$
f(x) x=f(e),
$$

that is

$$
f(x)=f(e) x^{-1}, \quad x \in G .
$$

Combining (91) and (92), we have

$$
\begin{gathered}
f(e)\left(f(e) y_{1}^{-1} \ldots f(e) y_{s}^{-1}\right)^{-1} f(e) y_{1}^{-1} \ldots f(e) y_{s-1}^{-1} \\
=f(e) y_{s} f(e)^{-1} \ldots y_{1} f(e)^{-1} f(e) y_{1}^{-1} \ldots f(e) y_{s-1}^{-1}=f(e) y_{s} f(e)^{-1}=y_{s}, \quad y_{s} \in G,
\end{gathered}
$$

which gives that $f(e) \in C$.

The proof is complete.

Proof of Theorem 15. We deal with only part (a), part (b) can be handled similarly.

Theorem 12 shows that if there is a neigbourhood $U \subset \mathbb{R}^{2}$ of $\left(a_{p}, a_{p}\right)$ such that equation (19) is globally $p$-periodic on $U$, then the homogeneous linear equation

$$
y(n)=\alpha y(n-1)+\beta y(n-2), \quad n \geq 0
$$

is globally $p$-periodic.

Lemma 21 can now be applied to equation (93): either $\beta=1$ and $\alpha=0$, and then $p=2$, or

$$
\beta=-1, \quad \text { and } \quad \alpha=2 \cos \left(\frac{2 \pi}{p} k\right), \quad 1 \leq k<\frac{p}{2} \quad \text { is an integer. }
$$

$\beta=1$ and $\alpha=0$ is impossible, by the conditions of the existence of a positive equilibrium point (see (20)).

In the second case $\alpha+\beta<1$, and hence $\gamma>0$ (see also (20)).

We have thus proved that the condition (22) is necessary. 
To prove that the condition (22) is sufficient, we first use Theorem 14 (b): since $a_{p}$ is an equilibrium point of the linear equation

$$
x(n)=\alpha x(n-1)+\beta x(n-2)+\gamma, \quad n \geq 0,
$$

(94) is globally $p$-periodic if and only if (93) is globally $p$-periodic. It follows that it is enough to prove the next: if (94) is globally $p$-periodic, then there is a neigbourhood $U \subset \mathbb{R}^{2}$ of $\left(a_{p}, a_{p}\right)$ such that every solution of (94) determined by an initial value $\varphi:=(\varphi(-2), \varphi(-1))$ from $U$ is a nonnegative sequence. Really, in this case every solution of equation (19) determined by an initial value from $U$ is a solution of (94) too.

Suppose that equation (94) is globally $p$-periodic. Since $x(n)=a_{p}(n \geq-2)$ is a solution of $(94)$, the solution $x(\cdot ; u, v)$ of $(94)$ determined by the initial value $(u, v) \in \mathbb{R}^{2}$ can be obtained as

$$
x(n ; u, v)=y\left(n ; u-a_{p}, v-a_{p}\right)+a_{p}, \quad n \geq-2,
$$

where $y(\cdot ; u, v)$ is the solution of $(93)$ determined by the initial value $(u, v)$. Thus we have to show that there exists a neigbourhood $V \subset \mathbb{R}^{2}$ of $(0,0)$ such that for every $(u, v) \in V$

$$
y(n ; u, v) \geq-a_{p}, \quad n \geq-2 .
$$

Because $y(\cdot ; u, v)$ is $p$-periodic, it is determined by the vector

$$
(u, v, y(0 ; u, v), \ldots, y(p-3 ; u, v)) \in \mathbb{R}^{p},
$$

and therefore (95) comes from

$$
y(n ; u, v) \geq-a_{p}, \quad-2 \leq n \leq p-3 .
$$

(93) is a homogeneous linear equation, and hence

$$
(u, v) \rightarrow(u, v, y(0 ; u, v), \ldots, y(p-3 ; u, v)), \quad(u, v) \in \mathbb{R}^{2}
$$

is a linear map, and thus it is continuous. This implies the result.

The proof is completed.

Proof of Theorem 17. It can be proved exactly the same way as Theorem 15.

Acknowledge: Supported by Hungarian National Foundations for Scientific Research Grant No. K101217.

\section{References}

[1] Raghib M. Abu-Saris, On the periodicity of the difference equation $x_{n+1}=$ $\alpha\left|x_{n}\right|+\beta x_{n-1}$, J. Difference. Eq. Appl. 5 (1999), pp. 57-69.

[2] Raghib M. Abu-Saris, A self-invertibility condition for global periodicity of difference equations, Appl. Math. Lett. 19 (2006), pp. 1078-1082. 
[3] Raghib M. Abu-Saris, and Q. M. Al-Hassan, On global periodicity of difference equations, J. Math. Anal. Appl. 283 (2003), pp. 468-477.

[4] M. Andreatta, and D. T. Vuza, On some properties of periodic sequences in Anatol Vireu's modal theory, Tatra Mt. Math. Publ. 23 (2001), pp. 1-15.

[5] F. Balibrea, A. Linero Bas, G. S. López, and S. Stević, Global periodicity of $x_{n+k+1}=f_{k}\left(x_{n+k}\right) \ldots f_{1}\left(x_{n+1}\right)$, J. Difference Equ. Appl. 13 (2007), pp. 901-910.

[6] L. Berg, Nonlinear difference equations with periodic solutions, Rostock. Math. Kolloq. 61 (2006), pp. 13-20.

[7] V. Botella-Soler, J. M. Castelo, J. A. Oteo, and J. Ros, Bifurcations in the Lozi map, J. Phys. A: Math. Theor. 44 (2011), pp. 14

[8] E. Camouzis, G. Ladas, F. Palladino, and E. P. Quinn, On the boundedness character of rational equations, part 1, J. Difference. Eq. Appl. 12 (2006), pp. 503-523.

[9] J. S. Canovas, A. Linero Bas, and G. Soler Lopez, On global periodicity of difference equations, Taiwanese J. Math. 13 (2009), pp. 1963-1983.

[10] A. Cima, A. Gasull, and V. Mańosa, Global periodicity conditions for maps and recurrences via normal forms, arXiv:1205.0923

[11] D. Clark, Periodic solutions of arbitrary length in a simple integer iteration, Adv. Difference Equ. (2006) 2006:035847, pp. 9

[12] S. Elaydi, An Introduction to Difference Equations, Springer-Verlag, New York, 1996.

[13] D. Dee, and M. Ghil, Boolean difference equations, I: Formulation and dynamic behavior, SIAM J. Appl. Math. 44 (1984), pp. 111-126.

[14] T. M. Flett, Differential Analysis, Cambridge University Press, Cambridge, 1980.

[15] M. I. Gil', S. Kang, and G. Zhang, Positive periodic solutions of abstract difference equations, Appl. Math. E-Notes 4 (2004), pp. 54-58.

[16] M. Ghil, and I. Zaliapin, A Novel Fractal Way: Boolean delay equations, and their applications to the geosciences, A BDE Review

[17] J. Greene, and K. Niedzielski, Periodic solutions to some difference equations over the integers, J. Difference. Eq. Appl. 16 (2010), pp. 321-346.

[18] E. A. Grove, G. Ladas, Periodicities in Nonlinear Difference Equations, Chapman \& Hall/CRC, 2005. 
[19] I. Győri, and L. Horváth, Sharp algebric periodicity conditions for linear higher order difference equations, Comput. Math. Appl. 64 (2012), pp. $2262-2274$.

[20] I. Győri, and L. Horváth, Utilization of the circulant matrix theory in periodic higher order autonomous difference equations, submitted

[21] I. S. Kotsireas, Homotopies and polynomial system solving I: Basic principles, ACM SIGSAM Bulletin 35 (2001), pp. 19-32.

[22] R. P. Kurshan, and B. Gopinath, Recursively generated periodic sequences, Can. J. Math. 26 (1974), pp. 1356-1371.

[23] G. Ladas, Difference equations with eventually periodic solutions, J. Difference. Eq. Appl. 2 (1996), pp. 97-99.

[24] J. Rubió-Massegú, and V. Mańosa, Normal forms for rational difference equations with applications to the global periodicity problem, J. Math. Anal. Appl. 332 (2007), pp. 896-918.

[25] R. J. Sacker, and H. F. von Bremen, Dynamic reduction with applications to mathematical biology and other areas, J. Biol. Dyn. 1 (2007), pp. 437-453.

[26] H. Sedaghat, Semiconjugate factorizations of higher order linear difference equations in rings, arXiv:1301.2804

[27] S. Semmes, An introduction to Heisenberg groups in analysis and geometry, Notices of the AMS 50 (2003), pp. 640-646.

[28] S. Stević, A note on periodic character of a higher order difference equation, Rostock. Math. Kolloq. 61 (2006), pp. 21-30.

[29] S. Stević, On global periodicity of a class of difference equations, Discrete Dyn. Nat. Soc. (2007) Art. ID 23503, pp. 10

[30] S. Tyler, and J. Loftsson, Periodic binary sequences with very good autocorrelation properties, TDA Progress Report (1985), pp. 48-82.

[31] G. Hoffmann de Visme, Binary Sequences, English University Press (London), 1971.

[32] B. Xia, and X. Hou, A complete algorithm for counting real solutions of polynomial systems of equations and inequalities, Comput. Math. Appl. 44 (2002), pp. 633-642. 